\title{
Testing and Modeling Multivariate Threshold Models
}

\author{
Ruey S. Tsay \\ Graduate School of Business \\ University of Chicago \\ Chicago, IL 60637 \\ rst@gsbrst.uchicago.edu
}

\begin{abstract}
Threshold autoregressive models in which the process is piecewise linear in the threshold space have received much attention in recent years. In this paper, we use predictive residuals to construct a test statistic to detect threshold nonlinearity in a vector time series and propose a procedure for building a multivariate threshold model. The thresholds and the model are selected jointly based on the Akaike information criterion. The finite-sample performance of the proposed test is studied by simulation. The modeling procedure is then used to study arbitrage in security markets and results in a threshold cointegration between logarithms of future contracts and spot prices of a security after adjusting for the cost-of-carrying the contracts. In this particular application, thresholds are determined in part by the transaction costs. We also apply the proposed procedure to U.S. monthly interest rates and two river flow series of Iceland.
\end{abstract}

Keywords: Akaike information criterion, Arranged autoregression, Model change, Nonlinearity test, Predictive residuals, Recursive least squares, Threshold cointegration. 


\section{Introduction}

To motivate the study of multivariate threshold models, we consider an application in finance in which an asset is traded simultaneously in two markets. Based on the theory of law of one price, the asset should have the same price at the same time in both markets; otherwise, one can buy the asset in the cheapest market and simultaneously sell it in the dearest market to make a profit. However, some minor deviation in prices of the asset may exist because other factors such as transaction cost, capital constraint, interest rate, and execution risk may discourage traders or market makers from trading if the potential profit is small. In other words, arbitrage opportunities occur only when the deviation in prices is substantial so that the potential profit exceeds the cost of trading. An arbitrage opportunity, however, cannot last long because advances in computer trading can easily take advantages of such an opportunity when it appears.

Let $p_{i t}$ be the log price of the asset in market $i$ at time $t$ and $z_{t}=p_{1 t}-p_{2 t}$ be the price differential in logarithms. Log-transformation is commonly used in financial data analysis. Then, $z_{t}$ cannot continuously assume large values in modulus, because there exist no long lasting arbitrage opportunities. From a statistical point of view, $z_{t+\ell}$ should have a high probability to return to its mean when $\left|z_{t}\right|$ is large. In finance, $z_{t}$ is said to be highly mean-reverting when $\left|z_{t}\right|$ is large. This behavior has profound impacts on the dynamics of $p_{i t}$ and we can formulate an approximate model for $p_{i t}$ as:

$$
\boldsymbol{p}_{t}= \begin{cases}f_{1}\left(\boldsymbol{p}_{t-1}, \boldsymbol{p}_{t-2}, \cdots ; \boldsymbol{\epsilon}_{1 t} \mid \boldsymbol{\theta}_{1}\right) & \text { if } z_{t-d} \leq r_{1} \\ f_{2}\left(\boldsymbol{p}_{t-1}, \boldsymbol{p}_{t-2}, \cdots ; \boldsymbol{\epsilon}_{2 t} \mid \boldsymbol{\theta}_{2}\right) & \text { if } r_{1}<z_{t-d} \leq r_{2} \\ f_{3}\left(\boldsymbol{p}_{t-1}, \boldsymbol{p}_{t-2}, \cdots ; \boldsymbol{\epsilon}_{3 t} \mid \boldsymbol{\theta}_{3}\right) & \text { if } r_{2}<z_{t-d},\end{cases}
$$

where $\boldsymbol{p}_{t}=\left(p_{1 t}, p_{2 t}\right)^{\prime}, f_{i}($.$) are well-defined functions such that f_{i}(.) \neq f_{j}($.$) for i \neq j, \boldsymbol{\theta}_{i}$ are finite-dimensional parameters, $\boldsymbol{\epsilon}_{i t}$ denote noise terms, $d$ is a positive integer denoting the average time taken to execute an arbitrage trade, and $r_{1}<0<r_{2}$ are real numbers. This model says that the dynamic behavior of $p_{i t}$ depends on the magnitude of $z_{t-d}$. When $z_{t-d}<r_{1}<0$, there exists an arbitrage opportunity of buying the asset at market 1 and selling simultaneously at market 2 . When $z_{t-d}>r_{2}>0$, an arbitrage opportunity occurs in the opposite direction. When $z_{t-d}$ is between $r_{1}$ and $r_{2}$, the price differential induces no arbitrage. The two prices should move closer to each other following any arbitrage trading.

Model (1) has three regimes and belongs to the class of general multivariate threshold models in which $z_{t}$ is referred to as the threshold variable, $d$ is the threshold lag or delay, and $r_{i}$ 's are the thresholds. See Tong (1990) and the references therein. In our particular example, the thresholds $r_{i}$ are functions of transaction cost, interest rate, economic risk, and financial purpose of a trade. If $p_{i t}$ are unit-root nonstationary, then model (1) represents a co-integrated system in which $z_{t}$ represents a stationary combination of the system, see Balke and Fomby (1997) for further discussions on threshold cointegration.

In this paper, we shall focus on linear models which may also depend on some exogenous variables. Consider a $k$-dimensional time series $\boldsymbol{y}_{t}=\left(y_{1 t}, \cdots, y_{k t}\right)^{\prime}$ and a $v$-dimensional exogenous variables $\boldsymbol{x}_{t}=\left(x_{1 t}, \cdots, x_{v t}\right)^{\prime}$. Let $-\infty=r_{0}<r_{1}<\cdots<r_{s-1}<r_{s}=\infty$. 
Then, $\boldsymbol{y}_{t}$ follows a multivariate threshold model with threshold variable $z_{t}$ and delay $d$ if it satisfies

$$
\boldsymbol{y}_{t}=\boldsymbol{c}_{j}+\sum_{i=1}^{p} \boldsymbol{\phi}_{i}^{(j)} \boldsymbol{y}_{t-i}+\sum_{i=1}^{q} \boldsymbol{\beta}_{i}^{(j)} \boldsymbol{x}_{t-i}+\boldsymbol{\epsilon}_{t}^{(j)} \quad \text { if } \quad r_{j-1}<z_{t-d} \leq r_{j}
$$

where $j=1, \cdots, s, \boldsymbol{c}_{j}$ are constant vectors, and $p$ and $q$ are non-negative integers. The innovations satisfy $\boldsymbol{\epsilon}_{t}^{(j)}=\boldsymbol{\Sigma}_{j}^{1 / 2} \boldsymbol{a}_{t}$, where $\boldsymbol{\Sigma}_{j}^{1 / 2}$ are symmetric positive definite matrices and $\left\{\boldsymbol{a}_{t}\right\}$ is a sequence of serially uncorrelated random vectors with mean zero and covariance matrix $\boldsymbol{I}$, the identity matrix. The threshold variable $z_{t}$ is assumed to be stationary and have a continuous distribution. Model (2) has $s$ regimes and is a piecewise linear model in the threshold space $z_{t-d}$, but it is nonlinear in time when $s>1$.

For model (2), we assume that the threshold variable $z_{t}$ is known, but the delay $d$, the number of regimes $s$, and the thresholds $r_{i}$ are unknown. Our goal is two-fold. First, we propose a test statistic to detect the need of employing such a model against the alternative of a linear model, i.e. $s=1$ versus $s>1$. The proposed test is a generalization of that in Tsay (1989) for the univariate case and has an asymptotic chi-square distribution. The test is simple, performs well in finite samples, yet it does not depend on the alternative model or encounter the problem of undefined parameters under the null hypothesis. The generalization also allows for exogenous variables and conditional heteroscedasticity. Second, we consider a procedure for building such a multivariate threshold model including estimation of $d$ and the thresholds. We employ the conditional least squares method in estimation and Akaike information criterion in model selection. The procedure is easier to apply than that of Tsay (1989) who uses scatterplots that often require subjective interpretations.

The paper is organized as follows. In Section 2, we consider an arranged regression analysis that transforms the threshold model in (2) into a change-point problem. We then use recursive least squares to obtain predictive residuals in the arranged regression and use the standardized predictive residual to construct the proposed test statistic. The asymptotic distribution of the proposed test is derived. We also use simulation to study the finite-sample performance of the proposed test. Some related literature is also reviewed. In Section 3, we briefly address conditional least squares estimation of the model. In Section 4 , we propose a procedure for model building. Akaike information criterion is used to select the thresholds as well as other parameters. The proposed test and modeling procedure are applied in Section 5 to two real examples of high-frequency data in finance. We compare the results with those available in the literature using other methods. The model we built appears to be in closer agreement with common expectations. Finally, we apply the modeling procedure to U.S. monthly interest rates and two daily river flow series of Iceland.

\section{Testing}

Consider the null hypothesis that $\boldsymbol{y}_{t}$ is linear versus the alternative hypothesis that it follows the multivariate threshold model in (2). This problem has attracted much attention in 
recent years, partly because of the difficulty that the thresholds $r_{i}$ are undefined under the null hypothesis when the likelihood ratio test is used. See Hansen (1996a), Chan and Tong (1990), and the references therein. Most of the likelihood-based tests consider the univariate case with two regimes, i.e. a single threshold, and use simulation to obtain critical values. On the other hand, Petruccelli and Davies (1986) and Tsay (1989) transform the testing problem into detecting change points using the concept of arranged autoregression and employ predictive residuals to construct test statistics that do not involve undefined parameters. More specifically, these tests use the threshold variable to construct an arranged regression, but do not depend on other features of the alternative model. They are simple and have familiar limiting distributions. For example, the test statistic of Tsay (1989) has an asymptotic F-distribution. Limited experience shows that this latter approach has decent power when the sample size is moderate or large. See Balke and Fomby (1997) for some power comparison.

In this section, we generalize the test statistic of Tsay (1989) to the multivariate model in (2) and use simulation to study the finite-sample performance of the test statistic. Several reasons justify such an extension. First, the test is extremely simple and has an asymptotic chi-square distribution. This is an important feature when the sample size is large such as in the analysis of high-frequency data in finance where the sample size can easily exceed 5000. Second, the test is widely applicable, including co-integrated systems. Its limiting distribution holds under weak regularity conditions. Third, the test has good power in detecting threshold nonlinearity.

\subsection{Arranged regression}

Given observations $\left\{\boldsymbol{y}_{t}, \boldsymbol{x}_{t}, z_{t}\right\}$, where $t=1, \cdots, n$, our goal is to detect the threshold nonlinearity of $\boldsymbol{y}_{t}$, assuming that $p, q$ and $d$ are known. To this end, we employ the least squares method and place the model in a regression framework:

$$
\boldsymbol{y}_{t}^{\prime}=\boldsymbol{X}_{t}^{\prime} \boldsymbol{\Phi}+\boldsymbol{\epsilon}_{t}^{\prime}, \quad t=h+1, \cdots, n
$$

where $h=\max (p, q, d), \boldsymbol{X}_{t}=\left(1, \boldsymbol{y}_{t-1}^{\prime}, \cdots, \boldsymbol{y}_{t-p}^{\prime}, \boldsymbol{x}_{t-1}^{\prime}, \cdots, \boldsymbol{x}_{t-q}^{\prime}\right)^{\prime}$ is a $(p k+q v+1)$-dimensional regressor, $\boldsymbol{\Phi}$ denotes the parameter matrix, and the notation $\boldsymbol{u}^{\prime}$ denotes the transpose of $\boldsymbol{u}$. If the null hypothesis holds, the least squares estimates of (3) are useful. On the other hand, the estimates are biased under the alternative hypothesis.

Equation (3) remains informative under the alternative hypothesis provided that we rearrange the ordering of the setup. For equation (3), the threshold variable $z_{t-d}$ assumes values in $S=\left\{z_{h+1-d}, \cdots, z_{n-d}\right\}$. Consider the order statistics of $S$ and denote the $i$-th smallest element of $S$ by $z_{(i)}$. Furthermore, let $t(i)$ be the time index of $z_{(i)}$. Then, the arranged regression based on the increasing order of the threshold variable $z_{t-d}$ is

$$
\boldsymbol{y}_{t(i)+d}^{\prime}=\boldsymbol{X}_{t(i)+d}^{\prime} \boldsymbol{\Phi}+\boldsymbol{\epsilon}_{t(i)+d}^{\prime}, \quad i=1, \cdots, n-h .
$$

It is important to see that in (4) the dynamic of the $\boldsymbol{y}_{t}$ series is not changed, that is, the independent variable of $\boldsymbol{y}_{t}$ is $\boldsymbol{X}_{t}$ for all $t$. What's changed is the ordering by which data 
enter the regression setup, that is, the row order if one places the regression in a matrix framework. An important feature of the arranged regression is that it effectively transforms a threshold model into a change-point problem, because the regression is arranged according to the increasing order of the threshold variable $z_{t-d}$.

\section{$2.2 \quad$ A test statistic}

There are many ways to detect model change in (4). Here we use predictive residuals and the recursive least squares method. Our idea is simple. If $\boldsymbol{y}_{t}$ is linear, then the recursive least squares estimator of the arranged regression (4) is consistent so that the predictive residuals approach white noise. Consequently, predictive residuals are uncorrelated with the regressor $\boldsymbol{X}_{t(i)+d}$. On the other hand, if $\boldsymbol{y}_{t}$ follows a threshold model, the predictive residuals would no longer be white noise because the least squares estimator is biased. In this case, the predictive residuals would be correlated with the regressor $\boldsymbol{X}_{t(i)+d}$.

Let $\hat{\boldsymbol{\Phi}}_{m}$ be the least squares estimate of $\boldsymbol{\Phi}$ of equation (4) with $i=1, \cdots, m$. That is, the estimate of arranged regression using data points associated with the $m$ smallest values of $z_{t-d}$. Let

$$
\begin{aligned}
\hat{\boldsymbol{e}}_{t(m+1)+d} & =\boldsymbol{y}_{t(m+1)+d}-\hat{\boldsymbol{\Phi}}_{m}^{\prime} \boldsymbol{X}_{t(m+1)+d} \\
\hat{\boldsymbol{\eta}}_{t(m+1)+d} & =\hat{\boldsymbol{e}}_{t(m+1)+d} /\left[1+\boldsymbol{X}_{t(m+1)+d}^{\prime} \boldsymbol{V}_{\boldsymbol{m}} \boldsymbol{X}_{t(m+1)+d}\right]^{1 / 2},
\end{aligned}
$$

where $\boldsymbol{V}_{m}=\left[\sum_{i=1}^{m} \boldsymbol{X}_{t(i)+d} \boldsymbol{X}_{t(i)+d}^{\prime}\right]^{-1}$, be the predictive residual and standardized predictive residual of regression (4), respectively. These quantities can be obtained efficiently by the recursive least squares algorithm. Next, consider the regression

$$
\hat{\boldsymbol{\eta}}_{t(\ell)+d}^{\prime}=\boldsymbol{X}_{t(\ell)+d}^{\prime} \Psi+\boldsymbol{w}_{t(\ell)+d}^{\prime}, \quad \ell=m_{o}+1, \cdots, n-h
$$

where $m_{o}$ denotes the starting point of the recursive least squares estimation. The problem of interest is then to test the hypothesis $H_{0}: \Psi=0$ versus the alternative $H_{a}: \Psi \neq \boldsymbol{O}$ in regression (7). Here we employ the test statistic

$$
C(d)=\left[n-h-m_{o}-(k p+v q+1)\right]\left\{\ln \left[\operatorname{det}\left(\boldsymbol{S}_{0}\right)\right]-\ln \left[\operatorname{det}\left(\boldsymbol{S}_{1}\right)\right]\right\}
$$

where the delay $d$ signifies that the test depends on the threshold variable $z_{t-d}, \operatorname{det}(\boldsymbol{A})$ denotes the determinant of the matrix $\boldsymbol{A}$, and

$$
\boldsymbol{S}_{0}=\frac{1}{n-h-m_{o}} \sum_{\ell=m_{o}+1}^{n-h} \hat{\boldsymbol{\eta}}_{t(\ell)+d} \hat{\boldsymbol{\eta}}_{t(\ell)+d}^{\prime}, \quad \boldsymbol{S}_{1}=\frac{1}{n-h-m_{o}} \sum_{\ell=m_{o}+1}^{n-h} \hat{\boldsymbol{w}}_{t(\ell)+d} \hat{\boldsymbol{w}}_{t(\ell)+d}^{\prime}
$$

where $\hat{\boldsymbol{w}}_{t}$ is the least squares residual of regression (7). Under the null hypothesis that $\boldsymbol{y}_{t}$ is linear and some regularity conditions, $C(d)$ is asymptotically a chi-square random variable with $k(p k+q v+1)$ degrees of freedom.

Remark 1: The null hypothesis of $\Psi=0$ includes a zero intercept for all predictive residuals. In theory, a non-zero intercept signifies a systematic bias in the estimates of 
arranged regression, indicating possible model changes. However, due to the possibility of finite-sample bias, one may wish in some applications to exclude the intercept terms from nonlinearity test in (8). In this case, $\boldsymbol{S}_{0}$ should be mean-corrected and the resulting test has an asymptotical chi-square distribution with $k(p k+q v)$ degrees of freedom.

For simplicity, when $\boldsymbol{y}_{t}$ is linear, we write the model as

$$
\boldsymbol{y}_{t}=\boldsymbol{c}+\sum_{i=1}^{p} \boldsymbol{\phi}_{i} \boldsymbol{y}_{t-i}+\sum_{i=1}^{q} \boldsymbol{\beta}_{i} \boldsymbol{x}_{t-i}+\boldsymbol{\epsilon}_{t}
$$

with $\boldsymbol{\epsilon}_{t}=\left(\epsilon_{1 t}, \cdots, \epsilon_{k t}\right)^{\prime}$. We shall assume that $\boldsymbol{c}=\mathbf{0}$ if the determinant $\left|\boldsymbol{I}-\boldsymbol{\phi}_{1} B-\cdots-\boldsymbol{\phi}_{p} B^{p}\right|$ has a zero on the unit circle. Let $\boldsymbol{V}_{n}$ be the usual $X^{\prime} X$-matrix of model (9) with $t=$ $1, \cdots, n$ and denote the minimum and maximum eigenvalues of $\boldsymbol{V}_{n}$ by $\lambda_{\min }(n)$ and $\lambda_{\max }(n)$, respectively. Then, the following theorem of Lai and Wei (1982, Theorem 1) establishes the strong consistency of the least squares estimates.

Theorem 1 Consider model (9) where $\boldsymbol{c}=\mathbf{0}$ if $\boldsymbol{y}_{t}$ contains any unit root. Suppose that $\left\{\boldsymbol{\epsilon}_{t}\right\}$ is a martingale difference sequence with respect to an increasing sequence of $\sigma$-fields $\left\{F_{t}\right\}$ such that

$$
\sup _{i, t} E\left(\left|\epsilon_{i t}\right|^{\alpha} \mid F_{t-1}\right)<\infty \quad \text { a.s. for some } \quad \alpha>2 .
$$

Furthermore, assume that $\left(\boldsymbol{y}_{t-1}, \cdots, \boldsymbol{y}_{t-p}, \boldsymbol{x}_{t-1}, \cdots, \boldsymbol{x}_{t-q}\right)$ is $F_{t-1}$-measurable such that

$$
\lambda_{\min }(n) \rightarrow \infty \quad \text { a.s. } \quad \text { and } \quad \ln \left[\lambda_{\max }(n)\right]=o\left(\lambda_{\min }(n)\right) \quad \text { a.s. }
$$

Then, the least squares estimates of equation (9) converge to $\boldsymbol{c}, \boldsymbol{\phi}_{i}$ and $\boldsymbol{\beta}_{i}$ a.s.

Theorem 2 Suppose that $\boldsymbol{y}_{t}$ follows the linear model in (9) and satisfies the conditions of Theorem 1. Moreover, assume that

$$
\operatorname{Cov}\left(\boldsymbol{\epsilon}_{t} \mid F_{t-1}\right)=\Sigma, \quad \text { a.s. }
$$

and $m_{o} \rightarrow \infty, n^{-1} m_{o} \rightarrow 0$ as $n \rightarrow \infty$. Then, the test statistic $C(d)$ of (8) follows asymptotically a chi-square distribution with $k(k p+v q+1)$ degrees of freedom for a fixed positive integer $d$, where $k$ and $v$ are the dimensional of $\boldsymbol{y}_{t}$ and $\boldsymbol{x}_{t}$, respectively.

Proof. By Theorem 1 and the condition of $m_{o}$, the standardized residuals $\hat{\boldsymbol{\eta}}_{t}$ converge a.s. to a martingale difference sequence, which under the assumption (11) is homogeneous. By the functional central limit theorem (Helland, 1982, Theorem 3.3), $\left(n-h-m_{o}\right) \boldsymbol{S}_{0}$ and $\left(n-h-m_{o}\right) \boldsymbol{S}_{1}$ of equation (8) follow asymptotically Wishart distributions. The result then follows the same argument as that in the multivariate multiple regression analysis, e.g. Johnson and Wichern (1988, p.308). $\square$.

Remark2: The homogeneity of $\boldsymbol{\epsilon}_{t}$ can be relaxed by modifying the standardization of the predictive residuals in the recursive least squares estimation. In particular, if $\boldsymbol{\epsilon}_{t}$ has 
conditional heteroscedasticity, then equation (6) no longer holds, but the $j$-th element of $\hat{\boldsymbol{\eta}}_{t(m+1)+d}$ can be obtained by the standardization

$$
\hat{\eta}_{j, t(m+1)+d}=\hat{e}_{j, t(m+1)+d} /\left[\hat{\sigma}_{j}^{2}+\boldsymbol{X}_{t(m+1)+d}^{\prime} \boldsymbol{V}_{m}^{*} \boldsymbol{X}_{t(m+1)+d}\right]^{1 / 2}
$$

where $\hat{\sigma}_{j}^{2}=\sum_{i=1}^{m} \hat{e}_{j, t(i)+d}^{2} /(m-k p-v q-1)$ is the residual mean square errors of the $j$-th element of $\boldsymbol{y}_{t}$ and

$$
\boldsymbol{V}_{m}^{*}=\boldsymbol{V}_{m}\left(\sum_{i=1}^{m} e_{j, t(i)+d}^{2} \boldsymbol{X}_{t(i)+d}^{\prime} \boldsymbol{X}_{t(i)+d}\right) \boldsymbol{V}_{m}, \quad \text { with } \quad \boldsymbol{V}_{m}=\left(\sum_{i=1}^{m} \boldsymbol{X}_{t(i)+d}^{\prime} \boldsymbol{X}_{t(i)+d}\right)^{-1}
$$

For conditional heteroscedastic models, $e_{j, t}^{2}$ is correlated with elements of $\boldsymbol{X}_{t}^{\prime} \boldsymbol{X}_{t}$ and the variances of the least squares estimates must be modified accordingly.

\subsection{Simulation}

We conduct a simulation study to examine the finite-sample performance of the proposed test statistic $C(d)$ in (8). For performance under the null hypothesis, we consider three models

$$
\begin{aligned}
& \boldsymbol{y}_{t}=\left[\begin{array}{rr}
0.7 & 0.2 \\
-0.2 & 0.7
\end{array}\right] \boldsymbol{y}_{t-1}+\boldsymbol{\epsilon}_{t}, \quad \boldsymbol{\Sigma}=\left[\begin{array}{ll}
1.0 & 0.3 \\
0.3 & 1.0
\end{array}\right] \\
& \boldsymbol{y}_{t}=\left[\begin{array}{rr}
0.9 & 0 \\
0.2 & 0.9
\end{array}\right] \boldsymbol{y}_{t-1}+\boldsymbol{\epsilon}_{t}, \quad \boldsymbol{\Sigma}=\boldsymbol{I} \\
& \boldsymbol{y}_{t}=\boldsymbol{\phi}_{1} \boldsymbol{y}_{t-1}+\boldsymbol{\phi}_{2} \boldsymbol{y}_{t-2}+\boldsymbol{\epsilon}_{t}, \quad \boldsymbol{\Sigma}=\boldsymbol{I},
\end{aligned}
$$

where $\boldsymbol{\epsilon}_{t}$ are independent multivariate normal random variates with mean zero and covariance matrix $\Sigma, \boldsymbol{I}$ denotes the identity matrix, and

$$
\boldsymbol{\phi}_{1}=\left[\begin{array}{rrrr}
0.8 & 0.2 & 0.0 & 0.2 \\
0.33 & -0.8 & 0.33 & -1.2 \\
0.2 & -0.2 & 1.0 & -0.2 \\
-0.33 & 1.8 & -0.33 & 2.2
\end{array}\right], \quad \phi_{2}=\left[\begin{array}{rrrr}
-0.15 & 0.15 & 0.0 & 0.15 \\
0.3 & -0.09 & 0.3 & -0.06 \\
0.15 & -0.15 & 0.0 & -0.15 \\
-0.3 & 0.09 & -0.3 & 0.06
\end{array}\right]
$$

Models (12) and (13) are stationary but Model (14) represents a co-integrated system with two unit roots; see Engle and Yoo (1987). We use the latter to illustrate that Theorems 1 and 2 apply to unit-root nonstationary processes. However, to avoid small-sample bias of the least squares estimator for unit-root processes, we exclude the constant term from the nonlinearity test in (8) for model (14) and use a larger $m_{o}$ to start the recursive estimation. See Remark 1.

The sample sizes used are $n=150$ and 300, and the number of replications is 10,000. Table 1 shows the empirical percentiles of the test statistic $C(d)$ and those of the corresponding chi-square distributions. In the test, we assume $y_{1, t-d}$ is the threshold variable, $d \in\{1,2,3,4\}$. The choice of $m_{o}$ is also given in the table. As anticipated, the empirical 
distributions of the test statistic $C(d)$ are close to their asymptotic chi-square distributions and do not depend on the choice of the delay $d$. Our limited experience shows that for a unit-root nonstationary series a small $m_{o}$ may introduce bias in the empirical distribution of $C(d)$, resulting in larger empirical percentiles. We use $m_{o} \approx 5 \sqrt{n}$ for the unit-root series and $m_{o} \approx 3 \sqrt{n}$ for the stationary case, where $n$ is the sample size. These choices satisfy the condition of Theorem 2 and work well in our study. In an application, the choice of $m_{o}$ is a compromise between stable starting estimation and good power in testing (i.e. keeping a reasonable sample size for testing). Further, different values of $m_{o}$ can be used to investigate the sensitivity of the test result with respect to the selection.

To study the power of the test, we employ two 2-dimensional threshold autoregressive models

$$
\boldsymbol{y}_{t}= \begin{cases}\boldsymbol{\phi}_{1}^{(1)} \boldsymbol{y}_{t-1}+\boldsymbol{\epsilon}_{t}^{(1)} & \text { if } y_{1, t-1}<0.0 \\ \boldsymbol{\phi}_{1}^{(2)} \boldsymbol{y}_{t-1}+\boldsymbol{\epsilon}_{t}^{(2)} & \text { if } y_{1, t-1} \geq 0.0\end{cases}
$$

where

$$
\begin{gathered}
\boldsymbol{\phi}_{1}^{(1)}=\left[\begin{array}{ll}
0.7 & 0.0 \\
0.3 & 0.7
\end{array}\right], \boldsymbol{\Sigma}_{1}=\left[\begin{array}{ll}
1.0 & 0.2 \\
0.2 & 1.0
\end{array}\right], \boldsymbol{\phi}_{1}^{(2)}=\left[\begin{array}{rr}
-0.7 & 0.0 \\
-0.3 & -0.7
\end{array}\right], \boldsymbol{\Sigma}_{2}=\left[\begin{array}{rr}
1.0 & -0.3 \\
-0.3 & 1.0
\end{array}\right], \\
\boldsymbol{y}_{t}=\left\{\begin{array}{l}
\boldsymbol{\phi}_{1}^{(1)} \boldsymbol{y}_{t-1}+\boldsymbol{\epsilon}_{t} \text { if } y_{1, t-1}<-3.3 \\
\boldsymbol{\phi}_{1}^{(2)} \boldsymbol{y}_{t-1}+\boldsymbol{\epsilon}_{t} \text { if }-3.3 \leq y_{1, t-1}<3.3 \\
\boldsymbol{\phi}_{1}^{(3)} \boldsymbol{y}_{t-1}+\boldsymbol{\epsilon}_{t} \text { if } y_{1, t-1} \geq 3.3
\end{array}\right.
\end{gathered}
$$

where

$$
\boldsymbol{\phi}_{1}^{(1)}=\left[\begin{array}{rr}
-0.9 & 0.0 \\
0.2 & -0.9
\end{array}\right], \boldsymbol{\phi}_{1}^{(2)}=\left[\begin{array}{ll}
1.2 & 0.0 \\
0.0 & 0.6
\end{array}\right], \boldsymbol{\phi}_{1}^{(3)}=\left[\begin{array}{rr}
-0.8 & 0.0 \\
0.2 & 0.8
\end{array}\right], \boldsymbol{\Sigma}=\boldsymbol{I} .
$$

Again, the innovations are independent multivariate normal with mean zero and variance $\boldsymbol{\Sigma}_{j}$. Table 2 gives the empirical probabilities of rejecting linearity using the critical value 12.59, which is the $5 \%$ significance level of a chi-square distribution with 6 degrees of freedom. The results are based on 10,000 replications of sample sizes 150 and 300. Furthermore, to see the stability of the results, the simulation is repeated three times. The starting point $m_{o}$ of the recursive least squares is 50 for the first simulation, which is approximately $3 \sqrt{300}$, and is 40 for the second and third simulations. The table also shows power of the test when the delay $d$ is misspecified. It is seen that the test has good power when the delay $d$ is correctly specified and the results are stable among the three simulations. The power of the test deteriorates when the specified delay moves away from the true $d$.

\section{Estimation}

In this section, we consider conditional least squares estimation of the threshold model in (2), assuming that $p, q$ and $s$ are known and the threshold variable $z_{t}$ is given. However, 
the delay $d$ and the thresholds are part of the parameters. Our goal is to generalize the results of Chan (1993) and Hansen (1996b) for the univariate case to model (2). For ease in presentation, we focus on the case of $s=2$ and write the model as

$$
\boldsymbol{y}_{t}= \begin{cases}\boldsymbol{X}_{t}^{\prime} \boldsymbol{\Phi}_{1}+\Sigma_{1}^{1 / 2} \boldsymbol{a}_{t} & \text { if } z_{t-d} \leq r_{1} \\ \boldsymbol{X}_{t}^{\prime} \boldsymbol{\Phi}_{2}+\Sigma_{2}^{1 / 2} \boldsymbol{a}_{t} & \text { if } z_{t-d}>r_{1}\end{cases}
$$

where $\boldsymbol{a}_{t}=\left(a_{1 t}, \cdots, a_{k t}\right)^{\prime}$. We assume that (a) $z_{t-d}$ is stationary and continuous with a positive density function $f(r)$ on a bounded subset of the real line, say $R_{o} \subset R$, and (b) $d \in\left\{1, \cdots, d_{o}\right\}$, where $d_{o}$ is a fixed positive integer. The parameters of model (17) are $\left(\boldsymbol{\Phi}_{1}, \boldsymbol{\Phi}_{2}, \boldsymbol{\Sigma}_{1}, \boldsymbol{\Sigma}_{2}, r_{1}, d\right)$, and their conditional least squares estimates can be obtained in two steps. First, for given $d$ and $r_{1}$, model (17) reduces to two separated multivariate linear regressions from which the least squares estimates of $\boldsymbol{\Phi}_{i}$ and $\boldsymbol{\Sigma}_{i}(i=1,2)$ are readily available. The estimates are

$$
\hat{\boldsymbol{\Phi}}_{i}\left(r_{1}, d\right)=\left(\sum_{t}^{(i)} \boldsymbol{X}_{t} \boldsymbol{X}_{t}^{\prime}\right)^{-1}\left(\sum_{t}^{(i)} \boldsymbol{X}_{t} \boldsymbol{y}_{t}^{\prime}\right), \quad \hat{\boldsymbol{\Sigma}}_{i}\left(r_{1}, d\right)=\frac{\sum_{t}^{(i)}\left(\boldsymbol{y}_{t}-\boldsymbol{X}_{t}^{\prime} \hat{\boldsymbol{\Phi}}_{i}^{*}\right)\left(\boldsymbol{y}_{t}-\boldsymbol{X}_{t}^{\prime} \hat{\boldsymbol{\Phi}}_{i}^{*}\right)^{\prime}}{n_{i}-k}
$$

where $\sum_{t}^{(i)}$ denotes summing over observations in regime $i, \hat{\mathbf{\Phi}}_{i}^{*}=\hat{\mathbf{\Phi}}_{i}\left(r_{1}, d\right), n_{i}$ is the number of data points in regime $i$, and $k$ is the dimension of $\boldsymbol{X}_{t}$, satisfying $k<n_{i}$, for $i=1,2$. Denote the sum of squares of residuals by

$$
S\left(r_{1}, d\right)=S_{1}\left(r_{1}, d\right)+S_{2}\left(r_{1}, d\right)
$$

where $S_{i}\left(r_{1}, d\right)$ denotes the trace of $\left(n_{i}-k\right) \hat{\Sigma}_{i}\left(r_{1}, d\right)$. In step 2 , the conditional least squares estimates of $r_{1}$ and $d$ are obtained by

$$
\left(\hat{r}_{1}, \hat{d}\right)=\operatorname{argmin}_{r_{1}, d} S\left(r_{1}, d\right)
$$

where $1 \leq d \leq d_{o}$ and $r_{1} \in R_{o}$. The resulting least squares estimates for the parameters in (18) are

$$
\hat{\mathbf{\Phi}}_{i}=\hat{\mathbf{\Phi}}_{i}\left(\hat{r}_{1}, \hat{d}\right), \quad \hat{\boldsymbol{\Sigma}}_{i}=\hat{\mathbf{\Sigma}}_{i}\left(\hat{r}_{1}, \hat{d}\right)
$$

Let $\operatorname{vec}(\boldsymbol{A})$ be the column stacking vector of the matrix $\boldsymbol{A}$ and define

$$
\begin{aligned}
\boldsymbol{D}(r) & =E\left(\boldsymbol{X}_{t} \boldsymbol{X}_{t}^{\prime} \mid z_{t-d}=r\right), \quad D_{2}(r)=E\left[\left(\boldsymbol{X}_{t}^{\prime} \boldsymbol{X}_{t}\right)^{2} \mid z_{t-d}=r\right] \\
\boldsymbol{V}_{i}(r) & =E\left(\boldsymbol{X}_{t} \boldsymbol{X}_{t}^{\prime} a_{i t}^{2} \mid z_{t-d}=r\right), \quad V_{2, i}(r)=E\left[\left(\boldsymbol{X}_{t}^{\prime} \boldsymbol{X}_{t}\right)^{2} a_{i t}^{4} \mid z_{t-d}=r\right] .
\end{aligned}
$$

Assumption 1 1. $\left(\boldsymbol{X}_{t}, z_{t-d}, \boldsymbol{a}_{t}\right)$ is strictly stationary with $\beta$-mixing coefficient $\beta_{j}=$ $O\left(j^{-\delta}\right)$, for some $\delta>4$;

2. $E\left(\boldsymbol{a}_{t} \mid F_{t-1}\right)=\mathbf{0}$, where $F_{t-1}$ is the $\sigma$-field generated by $\left(\boldsymbol{X}_{j+1}, z_{j+1-d}, \boldsymbol{a}_{j}\right)$ for $j \leq t-1$;

3. $E\left(\left|y_{i t}\right|^{4}\right)<\infty, E\left(\left|x_{j t}\right|^{4}\right)<\infty$, and $E\left(\left|a_{i t}\right|^{4}\right)<\infty$ for all $i$ and $j$; 
4. The density function $f(r)$ of $z_{t-d}$ is positive on a bounded subset $R_{o} \subset R$ and $r_{1}$ is an interior point of $R_{o}$;

5. $f(r), \boldsymbol{D}(r), D_{2}(r), \boldsymbol{V}_{i}(r)$ and $V_{2, i}(r)$ are continuous at $r=r_{1}$;

6. $\Delta \equiv \boldsymbol{\Phi}_{1}-\boldsymbol{\Phi}_{2} \neq \mathbf{0}$;

7. $\boldsymbol{\Delta}_{j}^{\prime} \boldsymbol{D}\left(r_{1}\right) \boldsymbol{\Delta}_{j}>0, \boldsymbol{\Delta}_{j}^{\prime} \boldsymbol{V}_{i}\left(r_{1}\right) \boldsymbol{\Delta}_{j}>0$ for $j=1, \cdots, k$, where $\boldsymbol{\Delta}_{j}$ is the $j$-th column of $\Delta$.

Then, following the same approaches as Chan (1993) and Hansen (1996b), we can establish asymptotic properties of the conditional least squares estimates for model (17).

Theorem 3 Consider model (17) and suppose that Assumption 1 holds. Then, the conditional least squares estimators are strongly consistent as the sample size increases. That is, $\hat{\boldsymbol{\Phi}}_{i} \rightarrow \boldsymbol{\Phi}_{i}, \hat{r}_{1} \rightarrow r_{1}, \hat{d} \rightarrow d$, and $\hat{\boldsymbol{\Sigma}}_{i} \rightarrow \boldsymbol{\Sigma}_{i}$ almost surely as the sample size $n$ goes to infinity. Furthermore, $\sqrt{n_{i}} \operatorname{vec}\left(\hat{\boldsymbol{\Phi}}_{i}-\mathbf{\Phi}_{i}\right)$ are asymptotically normal with mean zero and covariance matrix $\boldsymbol{\Gamma}_{i} \otimes \boldsymbol{\Sigma}_{i}$, where $\boldsymbol{\Gamma}_{i}=\lim _{n \rightarrow \infty}\left(\sum_{t}^{(i)} \boldsymbol{X}_{t} \boldsymbol{X}_{t}^{\prime}\right) / n_{i}$ and $\otimes$ denotes the Kronecker product.

In practice, $\boldsymbol{\Gamma}_{i}$ is estimated by $\left(\sum_{t}^{(i)} \boldsymbol{X}_{t} \boldsymbol{X}_{t}^{\prime}\right) / n_{i}$ and $\boldsymbol{\Sigma}_{i}$ by $\hat{\boldsymbol{\Sigma}}_{i}$.

Theorem 3 provides asymptotic results for multivariate threshold models in (2) for which the hyper-plane of the conditional expectation $E\left(\boldsymbol{y}_{t} \mid F_{t-1}\right)$ has a discontinuity at the threshold $z_{t-d}=r_{1}$, where $F_{t-1}$ denotes the information available at time $t-1$. Because of Assumption 1.7, it excludes the case in which the hyper-plane is continuous but not differentiable at the threshold. This latter case is much more involved. Chan and Tsay (1997) obtain the asymptotic result for the univariate continuous TAR model, but the result for the multivariate model is yet to be rigorously investigated. For processes satisfying Assumption 1.1, readers are referred to Pham and Tran (1985). Assumption 1.4 ensures that the sample size of each regime, i.e. $n_{i}$, goes to infinity when the sample size $n$ increases. Finally, for a limiting distribution of $\hat{r}_{1}$, see Hansen (1996b).

\section{$4 \quad$ Modeling}

Identifying an adequate multivariate threshold model for a given data set involves selection of many parameters. Except for the identification of the threshold variable $z_{t}$, the most difficult problem may be the specification of the number of regimes, i.e. the identification of $s$. In some applications, past experience and substantive information may provide useful information on the choice of $s$. In others, the computational complexity and the data may restrict $s$ to a small number such as 2 or 3 . In this section, we assume that $z_{t}$ and $s$ are given. When $s$ is unknown, we assume that it is either 2 or 3 and use some criterion statistics to make a selection. In an informal way, one may divide the data into subgroups according to the empirical percentiles of $z_{t-d}$ and use the test statistic (8) to detect any model change within each subgroup. This analysis can provide a preliminary estimate of $s$ and some possible locations for the thresholds. 
When $z_{t}$ and $s$ are given, we use the AIC criterion to select a model, assuming that $0 \leq p \leq p_{o} ; 0 \leq q \leq q_{o} ; 1 \leq d \leq d_{o}$. In some cases, one may use the test results of (8) for different $d$ to select the delay parameter, resulting in further simplification. This is based on the idea that the test is most powerful when $d$ is correctly specified. Given $p, q, d$ and $s$, the AIC criterion of a multivariate threshold model in (2) is

$$
A I C(p, q, d, s)=\sum_{j=1}^{s}\left[2 \ln \left(L_{j}(p, q, d, s)\right)+2 k(k p+v q+1)\right]
$$

where $L_{j}(p, q, d, s)$ is the likelihood function of regime $j$ evaluated at the maximum likelihood estimates of $\boldsymbol{c}_{j}, \boldsymbol{\phi}_{i}^{(j)}$ and $\boldsymbol{\beta}_{i}^{(j)}$. If the innovations are multivariate normal, then AIC reduces to

$$
A I C(p, q, d, s)=\sum_{j=1}^{s}\left[n_{j} \ln \left(\left|\hat{\boldsymbol{\Sigma}}_{j}\right|\right)+2 k(k p+v q+1)\right], \quad \text { with } \quad \hat{\boldsymbol{\Sigma}}_{j}=\frac{1}{n_{j}} \sum_{t}^{*} \hat{\boldsymbol{\epsilon}}_{t}^{(j)} \hat{\boldsymbol{\epsilon}}_{t}^{(j)^{\prime}}
$$

where $n_{j}$ is the number of data points in regime $j$, the summation $\sum_{t}^{*}$ is summing over observations in regime $j$, and $\hat{\boldsymbol{\epsilon}}_{t}^{(j)}$ are the residuals.

The AIC has been used in the literature to select threshold autoregressive models, see Tong (1990). When $p, q$ and $s$ are fixed, AIC is asymptotically equivalent to selecting the model that has the smallest generalized residual variance using the conditional least squares method.

\section{An Application}

As an illustration, we apply the multivariate threshold model to study index futures arbitrage in finance. See Forbes, Kalb and Kofman (1997), referred to as FKK hereafter, who consider a Bayesian analysis of this problem. We shall also discuss the differences between their model and the model we obtain. An arbitrage trading consists of simultaneously buying (short-selling) a security index and selling (buying) index futures of the same security whenever the prices diverge by more than the cost of carrying the security index over time until maturity of the futures contract. Here the cost involves transaction cost, dividend yields of the security, interest rate and many other factors. Some of the cost are known, but others are not. In the finance literature, Brenner and Kroner (1995) give the expression

$$
F_{t, T}-S_{t}=\left(r_{t, T}-q_{t, T}\right)(T-t)+z_{t}^{*}
$$

where $S_{t}$ is the logarithm of a security index price at time $t$ and $F_{t, T}$ is the logarithm of the index futures price at time $t$ with maturity $T, r_{t, T}$ is the risk-free interest rate, $q_{t, T}$ is the dividend yield on the security index, and $(T-t)$ is the time to maturity of the futures contract. For model $(22), z_{t}^{*}$ should be weakly stationary and, for arbitrage to be profitable, $z_{t}^{*}$ must exceed a certain value in modulus determined by transaction costs and other economic and risk factors. 
The data are the intra-day transaction data for the S\&P 500 stock index in May, 1993 and its June futures contract traded at the Chicago Mercantile Exchange. FKK used the data to construct a one-minute bivariate price series with 7060 observations. We use the same series. However, to avoid unduly influence of big outliers, we replaced 10 extreme values ( 5 on each side) by the simple average of their two nearest neighbors. This step does not affect the qualitative conclusion of our analysis.

The two series $F_{t, T}$ and $S_{t}$ contain a unit root. However, equation (22) indicates that they are co-integrated after adjusting the effect of interest rate and dividend yield. Consequently, the first differenced series with an error-correction term are used in the analysis. Let $f_{t}=F_{t, T}-F_{t-1, T}$ and $s_{t}=S_{t}-S_{t-1}$ be the return series and $z_{t}=100 z_{t}^{*}$. Figure 1 shows the time plot of $f_{t}, s_{t}$ and $z_{t}$. All three series fluctuate around a fixed mean and within a fixed range.

Let $\boldsymbol{y}_{t}=\left(f_{t}, s_{t}\right)^{\prime}$. The arbitrage theory and error-correction representation suggest a three-regime bivariate threshold model

$$
\boldsymbol{y}_{t}= \begin{cases}\boldsymbol{c}_{1}+\sum_{i=1}^{p} \boldsymbol{\phi}_{i}^{(1)} \boldsymbol{y}_{t-i}+\boldsymbol{\beta}_{1} z_{t-1}+\boldsymbol{\epsilon}_{t}^{(1)} & \text { if } z_{t-d} \leq r_{1} \\ \boldsymbol{c}_{2}+\sum_{i=1}^{p} \boldsymbol{\phi}_{i}^{(2)} \boldsymbol{y}_{t-i}+\boldsymbol{\beta}_{2} z_{t-1}+\boldsymbol{\epsilon}_{t}^{(2)} & \text { if } r_{1}<z_{t-d} \leq r_{2} \\ \boldsymbol{c}_{3}+\sum_{i=1}^{p} \boldsymbol{\phi}_{i}^{(3)} \boldsymbol{y}_{t-i}+\boldsymbol{\beta}_{3} z_{t-1}+\boldsymbol{\epsilon}_{t}^{(3)} & \text { if } r_{2}<z_{t-d}\end{cases}
$$

where $r_{1}<0<r_{2}$. The error-correction term can be $z_{t-\ell}$ for $1 \leq \ell \leq p$. We use $z_{t-1}$ as it is common in the literature. In the notation of model (2), we have $\boldsymbol{x}_{t}=z_{t-1}$ with $q=1$ and $v=1$.

FKK entertained model (23) with $p=8$. They used a Bayesian procedure with noninformative prior to estimate the model and obtained $\hat{d}=1$ with $\hat{r}_{1}=-.10381$ and $\hat{r}_{2}=$ 0.12763. They suggest that in this particular application, a trading order was typically executed within 4 minutes so that $\{1,2,3,4\}$ was used as possible values of $d$.

In our analysis, we begin with the multivariate threshold test of Section 2. Table 3(a) gives the test results of the statistic (8) for $d=\{1,2,3,4\}$ and $p=8$. The recursive estimation starts with $m_{o}=200$, which is about $2.5 \sqrt{7060}$. The null hypothesis is that the series are linear so that model (23) reduces to a bivariate linear model. The $p$-values of the test statistics, based on the asymptotic chi-square distribution with 36 degrees of freedom, are all close to zero. Therefore, as expected, the test strongly suggests threshold nonlinearity. The test also indicates that $d=1$ because it corresponds to the maximum of the test statistic. The test results continue to hold for all $p$ between 1 and 10 .

Turn to modeling. It is reasonable to assume that the prices are log-normal so that we use normal likelihood to evaluate the AIC criterion. For comparison purpose, we fixed $p=$ 8 so that the selection is only on the thresholds. Based on the empirical range of $z_{t-1}$, we assume that $r_{1} \in[-0.115,-0.02]$ and $r_{2} \in[.025,0.145]$. Using a grid search method with 300 points on each of the two intervals, the minimum AIC selects $\hat{d}=1$ with $\hat{r}_{1}=-0.022574$ and $\hat{r}_{2}=0.037673$. The minimum AIC is $-1.3099 \times 10^{5}$. The empirical thresholds obtained are very different from those of FKK. It is interesting to compare the two models.

In practice, arbitrage is only one of many possible reasons for trading the security indexes. But it is impossible to identify a trade that is purely for arbitrage purposes. Consequently, 
the empirical thresholds $\hat{r}_{i}$ of model (23) are not completely determined by transaction cost. For the S\&P 500 index, it seems that market-makers are more likely to take advantage of an arbitrage opportunity than individual investors. For market-makers, the transaction cost may be low.

\subsection{Comparison}

Table 4 gives the parameter estimates and their $t$-ratios of the model selected by the AIC criterion. Several interesting features are shown. First, as anticipated, the return series $f_{t}$ and $s_{t}$ do not depend on the co-integrated series $z_{t-1}$ in the middle regime. This is in agreement with common sense discussed in the Introduction. When the price difference is small, there is no arbitrage opportunity so that the two price series are not co-integrated. On the other hand, for the two outer regimes, the return series depend on the co-integrated series $z_{t-1}$; the $t$-ratios for the coefficients of $z_{t-1}$ are highly significant for returns of the S\&P500 stock index. On the contrary, the model of FKK fails to show this property. The $t$-ratios for the coefficients of $z_{t-1}$ for their middle regime are approximately 0.25 and 5.66, indicating some dependence on $z_{t-1}$. Second, the past returns of the futures series appear to be more informative in explaining the variations in both returns series, i.e. more $t$-ratios of $f_{t-i}$ are statistically significant. This is in agreement with the finding of FKK and the common knowledge that futures series are more liquid. Third, similar to that of FKK, our model also shows the negative first-order (partial) coefficient in futures price changes, that is, for the futures return $f_{t}$, the coefficients of $f_{t-1}$ is significantly negative in the lower regime and marginally negative in regimes 2 and 3. In finance, this phenomenon is referred to as the bid-ask spread induced bounce. Finally, the significance of the constant term in the outer regimes is understandable. For threshold models, these drift terms are needed to insure that the return series have zero unconditional means.

In summary, the model we obtained shows all the properties discussed in FKK that are in agreement with finance theory. In addition, our model also agrees with the common expectation of threshold co-integration discussed in Balke and Fomby (1997).

\subsection{Further analysis}

To better understand the system, we consider a univariate model for the co-integrated series $z_{t}$. Under the arbitrage argument, $z_{t}$ should follow a univariate self-exciting threshold autoregressive model with three regimes. The thresholds of this model should in theory be the same as those of the model for $\boldsymbol{y}_{t}$. Furthermore, the models in the outer regimes should have higher tendency in mean-reversion because there exists no prolonged arbitrage opportunity. Finally, the delay for $z_{t}$ should be the same as that of $\boldsymbol{y}_{t}$.

Table $3(\mathrm{~b})$ gives the results of applying the threshold test (8) to $z_{t}$ with $p=7$ and $q=$ 0 . The order 7 is obtained based on partial autocorrelation of $z_{t}$ and confirmed by the AIC criterion. The results clearly reject linearity and, as expected, confirm that the delay for $z_{t}$ is $d=1$. 
Next, we used AIC criterion to search for the thresholds. The thresholds are assumed to be in the intervals $[-0.12,-0.015]$ and $[0.02,0.145]$. With a grid search method using 300 points on each interval and $p=7$, the AIC criterion selects thresholds $\hat{r}_{1}=-0.017093$ and $\hat{r}_{2}=0.044086$ with minimum AIC equal to 4998.7. We further refine the search using intervals $[-0.04,-0.01]$ and $[0.02,0.06]$ and 200 grid points for each interval. The refinement does not yield reduction in the AIC criterion. Clearly, the selected thresholds for $z_{t}$ series are close to those for $\boldsymbol{y}_{t}$.

The parameter estimates of the model for $z_{t}$ are shown in Table 5. For simplicity by ignoring the marginally significant parameters in the outer regimes, the fitted model indeed confirms the expectation that $z_{t}$ has stronger mean-reverting tendency in the outer regimes. The estimates of the coefficients of $z_{t-1}$ are $0.81,0.93$, and 0.88 , respectively, for the three regimes. While the differences in coefficients are not large, they do match with common belief. In sum, the empirical threshold model for $z_{t}$ is in agreement with that for $\boldsymbol{y}_{t}$.

\subsection{Symmetric thresholds}

In this particular application, it maybe reasonable to assume that the two thresholds are symmetric with respect to zero, that is, $r_{1}=-r_{2}$ in model (23). Imposing symmetry substantially reduces the computation of threshold selection via the AIC criterion. For illustration and comparison, we assume $r_{2} \in[.02, .085]$ and use a grid search method with 700 points to select jointly the thresholds and the delay parameter. Table 6 shows the minimum AIC and the corresponding thresholds for $d \in\{1,2,3,4\}$. The overall AIC selects $\hat{d}=1$ with thresholds $r_{2}=-r_{1}=.03122$, which is reasonably close to the choices of asymmetric model. Figure 2 shows the scatterplot of AIC versus threshold $r_{2}$. The AIC is well behaved with a unique minimum. The fitted model with symmetric thresholds shows the same features as those given in Table 4. For example, the dependence of return series $f_{t}$ and $s_{t}$ on $z_{t-1}$ remains insignificant in the middle regime.

Finally, residual analysis of the threshold models built shows no significant serial correlations, but some minor conditional heteroscedasticity. The latter may be due to the occasional large values in the data; see Figure 1. Because the sample size is large, minor conditional heteroscedasticity should not alter the results obtained in our analysis.

\subsection{Another data set}

To double check the threshold estimation, we also apply the symmetric threshold model to a second data set, consisting of the same variables for November 1993 with contract expiration in December 1993. This new data set has 7693 observations. Again, we replace the 10 most extreme values by the averages of their two nearest neighbors. The proposed test again confirms threshold nonlinearity with $\hat{d}=1$. Specifically, with $p=10$ and $q=1$, the $C(d)$ statistics are $103.46,84.70,98.96$, and 106.28 , respectively, for $d=1, \cdots, 4$. The asymptotic distribution of these statistics is chi-square with 44 degrees of freedom. 
Using symmetric thresholds with $r_{2} \in[.015, .15]$ and 700 grid points, the AIC selects $\hat{r}_{2}$ $=0.040613$, which is close to 0.03122 obtained for the May data.

\section{More Applications}

\subsection{Analysis of U.S. Interest Rates}

As a second example, we consider U.S. monthly interest rates from 1959.1 to 1993.2. The series used are the three-month Treasury Bills and three-year Treasury Notes, representing short-term and intermediate series in the term-structure of interest rates. Denote the two interest-rate series by $Y_{1 t}$ and $Y_{2 t}$. We employ the growth series in our study, that is, we use $\boldsymbol{y}_{t}=\left(y_{1 t}, y_{2 t}\right)^{\prime}$, where $y_{i t}=\ln \left(Y_{i t}\right)-\ln \left(Y_{i, t-1}\right)$. Figure $3(\mathrm{a})$ and (b) show the time plots of $\boldsymbol{y}_{t}$. Each $y_{i t}$ series has 409 observations and fluctuates around a fixed level and within a fixed range, indicating weak stationarity.

Let $x_{t}=\ln \left(Y_{1 t}\right)-\ln \left(Y_{2 t}\right)$ be the "spread" in the logged interest rates. Under normal economic conditions, interest rates are positively correlated with maturities. The correlation between interest rates and maturities may become negative when the U.S. economy is in contraction (or recession). The latter situation is referred to as "inverted yield curve" in the economic literature. Consequently, $x_{t}$ assumed negative values more often than positive ones. The magnitude of $x_{t}$, therefore, may indicate the status of the U.S. economy, and it seems reasonable to use $x_{t}$ as a threshold variable in our analysis. However, to reduce random fluctuations in U.S. monthly interest rates and to incorporate the common knowledge that interest-rate changes were made carefully using perhaps quarterly economic information, we use a 3-month moving average of $x_{t}$ as the threshold variable. More specifically, the threshold variable $z_{t}$ is defined as follows:

$$
z_{1}=x_{1}, \quad z_{2}=\left(x_{2}+x_{1}\right) / 2, \quad z_{t}=\left(x_{t}+x_{t-1}+x_{t-2}\right) / 3, \quad t \geq 3,
$$

which is the 3-month "average spread" in logged interest rates. Figure 3(c) shows the time plot of $z_{t}$. As expected, $z_{t}$ only assumed positive values in the middle $70 \mathrm{~s}$ and early $80 \mathrm{~s}$ when the U.S. economy was weak.

Our analysis starts with threshold nonlinearity test. Under the null hypothesis of linearity, both the AIC criterion and the chi-square test of Tiao and Box (1981) select a bivariate $\operatorname{AR}(7)$ model for $\boldsymbol{y}_{t}$. Using $p=7$ and $z_{t}$ of (24) as the threshold variable, we perform the proposed test of Section 2 using different starting numbers of observations for the recursive least squares. The test results are given in Table 7 and clearly reject the linear hypothesis. The test statistics also suggest using delay $\hat{d}=4$ or $\hat{d}=1$, corresponding to delay by a quarter or a month. We re-run the test with recursive least squares starting at $m_{o}=60$ and 75 , respectively. The results confirm nonlinearity and possible values of $d$. Consequently, in our further analysis, we employ $d \in\{1,4\}$.

With 409 observations, $p=7$ and $d \in\{1,4\}$, we entertain the possibilities of multivariate TAR models with 2 or 3 regimes. Therefore, there are four combinations of $d$ and $s$, where $s$ 
is the number of regimes. For each combination of $d$ and $s$, we use a grid search method and AIC to select the thresholds. For 2-regime models, we assume the threshold $r_{1} \in[-0.30, .05]$ and employ 300 grid points. For 3 -regime models, we assume $r_{1} \in[-0.30,-0.20]$ and $r_{2} \in[-0.15, .05]$. The numbers of grid points are 70 and 50, respectively, for $r_{1}$ and $r_{2}$. The intervals are chosen based on the range of $z_{t}$. We summarize the results in Table 8 . From the table, it is clear that 3-regime models are preferred over 2-regime models. Between the two 3-regime models, $\hat{d}=4$ has a smaller AIC, but the difference is very small. In sum, $(\hat{p}, \hat{d}, \hat{s})=(7,4,3)$ is selected by AIC for the data.

Finally, there is no particular reason to assume that the AR order must be the same in all regimes. Indeed the estimation results with $p=7$ show many insignificant parameters. We further refine the model by using different AR orders for different regimes. Let $p_{i}$ be the AR order for the $i$-th regime. The AIC criterion selects the orders $\left(p_{1}, p_{2}, p_{3}\right)=(2,6,7)$ for the three regimes. Table 9 gives the conditional least squares estimates of the refined model. Residual analysis of the model indicates there are no significant serial correlations in the residuals or the squared residuals. From Table 9, we make the following observations. First, as anticipated, the growth series of interest rates are concurrently correlated. The concurrent sample correlations of the residuals are $0.74,0.73$ and 0.69 with asymptotic standard errors 0.085, 0.085 and 0.089, respectively, for the three regimes. Second, the dynamic structure of the two growth series depends on the status of U.S. economy. In regime 1, which presents economic expansion periods with 3-month interest rate much lower than 3-year rate, the two growth series behaved basically as uncoupled processes, even though each series shows some minor autocorrelations. Using observations in this regime only, the likelihood ratio test for zero off-diagonal elements in the AR matrices is 7.63 which corresponds to a $p$-value of 0.11 under the asymptotic $\chi_{4}^{2}$ distribution. In regime 2 , which represents moderate or stable economy, there exists a unidirectional relation from the short-term rates to the longer-term rates. The conclusion is supported by a likelihood ratio test of 6.51 , which gives a $p$-value of 0.37 under the asymptotic $\chi_{6}^{2}$ distribution. In regime 3, which represents economic slowdown or recession, feedback relation exists between the two growth series.

\subsection{Analysis of Iceland River Flow Data}

In this subsection, we demonstrate that the proposed multivariate threshold models are also applicable outside the realm of economics by considering briefly two daily river flow series of Iceland. The data are available in Tong (1990) who provided geographical and meteorological conditions of the rivers and analyzed the series individually. The dependent variables are the daily river flow, measured in $\mathrm{m}^{3} \mathrm{~s}^{-1}$, of Jökulsá Eystri River (denoted by $\left.y_{1 t}\right)$ and Vatnsdalsá River $\left(y_{2 t}\right)$ from 1972 to 1974 . The exogenous variables are daily precipitation $\left(x_{t}\right)$ in $\mathrm{mm}$ and temperature $\left(z_{t}\right)$ in ${ }^{\circ} \mathrm{C}$ collected at the meteorological station at Hveravellir. Following Tong (1990), the precipitation is shifted forward by one because the recorded value is the accumulated rain at $9 \mathrm{a} . \mathrm{m}$. from the same time the day before. There are 1095 observations after the shift in precipitation. Figure 4 shows the time plots 
of the four variables. Some nonlinear features of the river flow series such as sharp rises and slow declines are evident from the plots.

The strong nonlinearity of $\boldsymbol{y}_{t}=\left(y_{1 t}, y_{2 t}\right)^{\prime}$ is supported by the proposed threshold test in (8). Table 10 gives the test results for three bivariate AR models and three possible threshold variables. The exogenous variables used in the tests are lagged values of precipitation $\left(x_{t-1}, x_{t-2}, x_{t-3}\right)$ and contemporaneous and lagged values of temperature $\left(z_{t}, z_{t-1}\right)$. The threshold variables used are lagged values of the individual river flow and the contemporaneous and lagged values of daily temperature. We do not use precipitation as a threshold variable because the distribution of daily precipitation is not purely continuous. All of the tests are highly significant compared with their asymptotic chi-squared distributions; most of the $p$-values are close to zero. In fact, the significance of the tests is insensitive to the selection of $\mathrm{AR}$ order, ranging from $\mathrm{AR}(4)$ to $\mathrm{AR}(22)$ we have considered. Based on the highly significant test result and the fact that temperature influences snow melting that affects river flows, we select $z_{t}$ as the threshold variable. Furthermore, it seems logical to focus on two-regime TAR models in model selection for there is only a single freezing point.

Because temperatures in the drainage areas of the two rivers depend on the altitudes and distances from the sea, we use AIC and the conditional least squares method to refine the estimation of the threshold. Using 400 equally spaced grid points in the interval $\left[-10^{\circ} C, 6^{\circ} C\right]$ and the AIC criterion, we obtain $\hat{r}_{1}=-0.42394$ for all three vector AR models in Table 10. Figure 5 shows the scatterplot of AIC versus the possible threshold values for the AR(15) model. The selection is clear. The selected threshold is close to the freezing point and is in good agreement with the non-parametric estimation result showing in Figure 7.47 of Tong (1990, p.439) for the univariate relation between $y_{1 t}$ and $z_{t}$. Finally, we focus on the bivariate TAR(15) model and use AIC to select the AR order for each regime. The selected model is given in Table 11 with AIC $=16943.7$. The numbers of observations in each regime are 479 and 601, respectively, and the residual covariance matrices are

$$
\hat{\Sigma}_{1}=\left[\begin{array}{ll}
1.62 & 0.13 \\
0.13 & 0.46
\end{array}\right], \quad \hat{\Sigma}_{2}=\left[\begin{array}{cc}
47.83 & 2.40 \\
2.40 & 5.97
\end{array}\right]
$$

Residual analysis indicates that the fitted model is adequate with no strong serial correlations in the residuals. But a few clusters of large residuals remain, suggesting the possibility of minor periodic behavior in the river flow caused by seasonality. For comparison, the AIC for a bivariate linear AR(15) model using the same exogenous variables is 20422.8.

The model in Table 11 has some interesting features. First, the models in the second regime have larger innovational variances. This is not surprising because the second regime consists of days with temperature greater than $-0.42394^{\circ} C$, which include days with rain and snow melting in Iceland. Second, the model shows a feedback relation between the two rivers. We interpret this relationship as an indication of missing useful variables such as observations of evaporation and ground moisture content. These missing variables can influence the effects of precipitation on river flow. Third, as expected, the effects of precipitation on river flows are more pronounced in the second regime; see the larger $t$-ratios of the coefficients of $x_{t-i}$. 


\section{Concluding Remarks}

In this paper, we proposed a test statistic for detecting threshold nonlinearity in a vector time series and a procedure for building multivariate threshold models. We illustrated the proposed test and procedure via analysis of high-frequency financial data, monthly U.S. interest rates and two daily river flow series of Iceland. The models obtained are in agreement with common expectation.

Many problems remain open for the multivariate threshold models. For example, the sufficient and necessary conditions for stationarity of the model are largely unknown (see a special case in Chan, et al. (1985)) and the search for an appropriate threshold variable $z_{t}$ in an application needs a careful investigation.

Acknowledgement: This research is supported by the National Science Foundation. and the Graduate School of Business, University of Chicago. The author wishes to thank K.S. Chan, Jeff Russell and two referees for their helpful comments. 


\section{References}

Balke, N. S. and Fomby, T.B. (1997), "Threshold cointegration," International Economic Review, 38, 627-645.

Brenner, R. J. and Kroner, K. F. (1995), "Arbitrage, cointegration, and testing the unbiasedness hypothesis in financial markets," Journal of Financial and Quantitative Analysis, 30, 23-42.

Chan, K.S. (1993) "Consistency and limiting distribution of the least squares estimator of a threshold autoregressive model," Annals of Statistics, 21, 520-533.

Chan, K.S., Petruccelli, J.D., Tong, H., and Woolford, S.W. (1985), "A multiple threshold AR(1) model," Journal of Applied Probability, 22, 267-279.

Chan, K.S. and Tong, H. (1990), "On likelihood ratio tests for threshold autoregression," Journal of Royal Statistical Society, Series B, 52, 469-476.

Chan, K.S. and Tsay, R.S. (1997), "Limiting properties of the least squares estimator of a continuous threshold autoregressive model," Biometrika (to appear).

Engle, R.F. and Yoo, S. (1987), "Forecasting and testing in a co-integrated system," Journal of Econometrics, 35, 143-159.

Forbes, C.S., Kalb, G.R.J. and Kofman, P. (1997), "Bayesian arbitrage threshold analysis," Working Paper, Department of Economics, Monash University, Australia.

Hansen, B. E. (1996a), "Inference when a nuisance parameter is not identified under the null hypothesis," Econometrica, 64-413-430.

Hansen, B. E. (1996b), "Sample splitting and threshold estimation," Working paper, Department of Economics, Boston College.

Helland, I.S. (1982), "Central limit theorems for martingales with discrete or continuous time," Scandinavian Journal of Statististics, 9, 79-94.

Lai, T.L. and Wei, C.Z. (1982), "Least squares estimates in stochastic regression models with applications to identification and control of dynamic systems," Annals of Statistics, 10, 154-166.

Johnson, R.A. and Wichern, D.W. (1988), Applied Multivariate Statistical Analysis, 2nd edition, Prentice-Hall: Englewood Cliffs, New Jersey.

Petruccelli, J. and Davies, N. (1986), "A Portmanteau test for self-exciting threshold autoregressive-type nonlinearity in time series," Biometrika, 73, 687-694. 
Pham, T.D. and Tran, L.T. (1985), "Some mixing properties of time series models," Stochastic Processes and Their Applications, 19, 297-303.

Tiao, G. C. and Box, G. E. P. (1981), "Modeling multiple time series with applications," Journal of the American Statistical Association, 76, 802-816.

Tong, H. (1990), Nonlinear Time Series: A Dynamical System Approach, Oxford: Oxford University Press.

Tsay, R.S. (1989), "Testing and modeling threshold autoregressive processes," Journal of the American Statistical Association, 84, 231-240. 
(a) First differenced In(future)

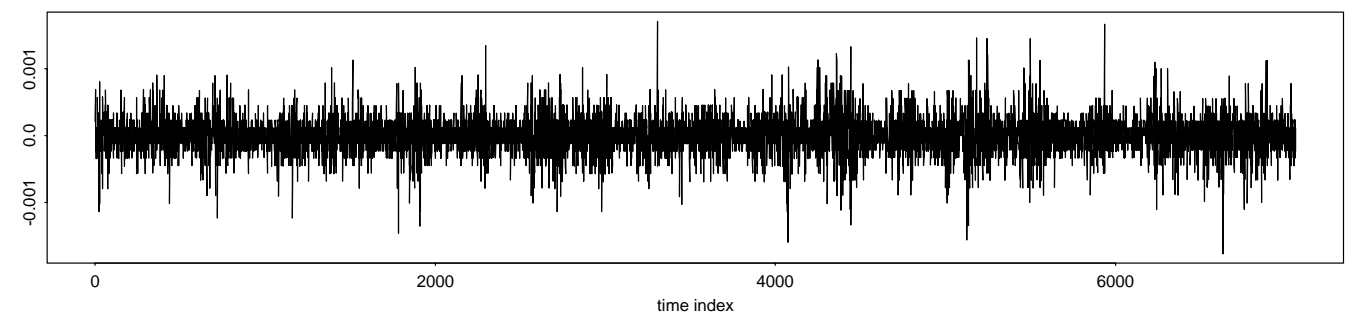

(b) First differenced In(price)
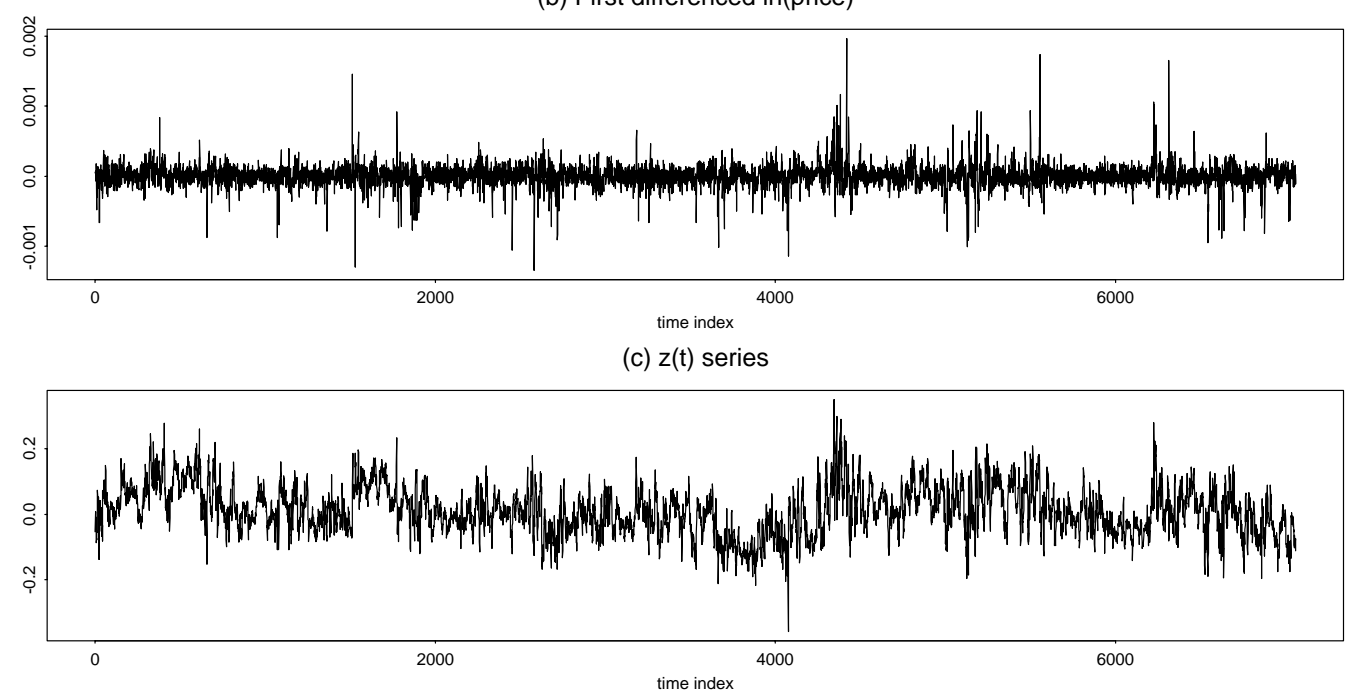

Figure 1: Time plots of minute-returns of S\&P500 index futures and prices and the associated threshold variable, May 1993 
Table 1: Empirical distribution of the test statistic $C(d)$ under the null hypothesis of linear vector models with $z_{t-d}=y_{1, t-d}$ and the recursive estimation starts at $m_{o}$. The results are based on 10,000 replications.

\begin{tabular}{|c|c|c|c|c|c|c|c|c|c|c|c|}
\hline Prob & .005 & .01 & .025 & .05 & .1 & .5 & .9 & .95 & .975 & .99 & .995 \\
\hline$d$ & \multicolumn{11}{|c|}{ (a) Model (12), $n=150, m_{o}=40$} \\
\hline 1 & 0.67 & 0.88 & 1.24 & 1.62 & 2.19 & 5.30 & 10.51 & 12.47 & 14.34 & 16.53 & 18.23 \\
\hline 2 & 0.64 & 0.84 & 1.20 & 1.59 & 2.19 & 5.23 & 10.69 & 12.65 & 14.66 & 17.00 & 18.72 \\
\hline 3 & 0.67 & 0.86 & 1.20 & 1.61 & 2.17 & 5.41 & 10.75 & 12.65 & 14.62 & 17.00 & 18.34 \\
\hline 4 & 0.67 & 0.86 & 1.22 & 1.65 & 2.24 & 5.41 & 10.70 & 12.68 & 14.51 & 17.04 & 18.97 \\
\hline$d$ & \multicolumn{11}{|c|}{ (b) Model (12), $n=300, m_{o}=50$} \\
\hline 1 & 0.67 & 0.86 & 1.18 & 1.61 & 2.17 & 5.29 & 10.45 & 12.38 & 14.30 & 16.91 & 18.36 \\
\hline 2 & 0.65 & 0.85 & 1.18 & 1.60 & 2.19 & 5.27 & 10.55 & 12.42 & 14.22 & 16.22 & 17.53 \\
\hline 3 & 0.69 & 0.88 & 1.24 & 1.63 & 2.21 & 5.34 & 10.72 & 12.64 & 14.52 & 16.64 & 18.58 \\
\hline 4 & 0.72 & 0.92 & 1.24 & 1.62 & 2.19 & 5.33 & 10.57 & 12.46 & 14.46 & 16.95 & 18.55 \\
\hline$d$ & \multicolumn{11}{|c|}{ (c) Model (13), $n=150, m_{o}=40$} \\
\hline 1 & 0.78 & 0.94 & 1.30 & 1.67 & 2.27 & 5.53 & 11.01 & 12.88 & 14.70 & 16.93 & 18.55 \\
\hline 2 & 0.68 & 0.85 & 1.23 & 1.64 & 2.22 & 5.36 & 10.74 & 12.80 & 14.71 & 16.99 & 18.32 \\
\hline 3 & 0.69 & 0.87 & 1.27 & 1.65 & 2.21 & 5.36 & 10.91 & 12.93 & 14.93 & 17.31 & 18.87 \\
\hline 4 & 0.69 & 0.95 & 1.28 & 1.67 & 2.25 & 5.47 & 11.02 & 13.16 & 15.02 & 17.47 & 19.20 \\
\hline$d$ & \multicolumn{11}{|c|}{ (d) Model (13), $n=300, m_{o}=50$} \\
\hline 1 & 0.75 & 0.94 & 1.28 & 1.68 & 2.24 & 5.35 & 10.60 & 12.54 & 14.25 & 16.49 & 18.61 \\
\hline 2 & 0.67 & 0.92 & 1.27 & 1.63 & 2.20 & 5.24 & 10.51 & 12.55 & 14.34 & 16.58 & 18.17 \\
\hline 3 & 0.69 & 0.91 & 1.26 & 1.66 & 2.21 & 5.32 & 10.57 & 12.64 & 14.79 & 17.11 & 19.30 \\
\hline 4 & 0.68 & 0.90 & 1.23 & 1.59 & 2.18 & 5.34 & 10.65 & 12.94 & 14.63 & 17.25 & 18.92 \\
\hline$\chi_{6}^{2}$ & .68 & .87 & 1.24 & 1.64 & 2.20 & 5.35 & 10.64 & 12.59 & 14.45 & 16.81 & 18.55 \\
\hline$d$ & \multicolumn{11}{|c|}{ (e) Model (14), $n=150, m_{o}=60$} \\
\hline 1 & 15.45 & 16.49 & 18.72 & 20.66 & 22.84 & 32.04 & 43.46 & 47.26 & 50.85 & 54.94 & 57.26 \\
\hline 2 & 15.31 & 16.70 & 18.68 & 20.45 & 22.66 & 31.98 & 43.34 & 47.14 & 50.46 & 54.07 & 56.74 \\
\hline 3 & 15.33 & 16.63 & 18.59 & 20.49 & 22.76 & 32.03 & 43.30 & 47.06 & 50.28 & 54.90 & 57.84 \\
\hline 4 & 15.49 & 16.70 & 18.70 & 20.57 & 22.70 & 32.06 & 43.25 & 46.83 & 49.92 & 53.88 & 57.00 \\
\hline$d$ & \multicolumn{11}{|c|}{ (f) Model (14), $n=300, m_{o}=90$} \\
\hline 1 & 15.28 & 16.51 & 18.36 & 20.11 & 22.38 & 31.32 & 42.53 & 46.30 & 49.64 & 53.25 & 56.32 \\
\hline 2 & 15.26 & 16.56 & 18.43 & 20.05 & 22.32 & 31.26 & 42.68 & 46.03 & 49.15 & 53.69 & 56.14 \\
\hline 3 & 15.39 & 16.28 & 18.36 & 20.09 & 22.30 & 31.29 & 42.44 & 46.24 & 49.48 & 53.55 & 56.56 \\
\hline 4 & 15.42 & 16.55 & 18.29 & 20.07 & 22.29 & 31.19 & 42.49 & 45.90 & 49.13 & 53.25 & 56.16 \\
\hline$\chi_{32}^{2}$ & 15.13 & 16.36 & 18.29 & 20.07 & 22.27 & 31.34 & 42.58 & 46.19 & 49.48 & 53.49 & 56.33 \\
\hline
\end{tabular}


Table 2: Empirical probability of rejecting linearity when the process follows a vector TAR model, using $5 \%$ asymptotic critical value. The simulation is repeated three times.

\begin{tabular}{|l|r|rrr|r|rrr|}
\hline & \multicolumn{4}{|c|}{$n=150$} & \multicolumn{4}{c|}{$n=300$} \\
\hline & true $d$ & \multicolumn{2}{|c|}{ Misspecified $d$} & true $d$ & \multicolumn{3}{|c|}{ Misspecified $d$} \\
\hline Model & 1 & 2 & 3 & 4 & 1 & 2 & 3 & 4 \\
\hline$(15)$ & 99.4 & 46.2 & 23.2 & 16.9 & 100.0 & 80.3 & 43.9 & 25.6 \\
& 99.4 & 45.3 & 22.4 & 15.4 & 100.0 & 79.8 & 43.2 & 26.0 \\
& 99.3 & 44.9 & 22.7 & 15.9 & 100.0 & 79.1 & 44.5 & 25.6 \\
\hline$(16)$ & 87.7 & 34.3 & 25.4 & 18.5 & 97.9 & 66.8 & 43.3 & 29.6 \\
& 87.9 & 33.3 & 25.1 & 17.9 & 98.2 & 66.7 & 42.8 & 30.1 \\
& 87.9 & 33.6 & 24.6 & 18.1 & 98.0 & 67.1 & 42.9 & 29.9 \\
\hline
\end{tabular}

Table 3: Results of threshold nonlinearity test. The sample size is 7060 and the starting point of recursive least squares is 200 .

\begin{tabular}{|c|rrrr|rrrr|}
\hline & \multicolumn{9}{|c|}{ (a) Bivariate, $p=8, q=1$} & \multicolumn{4}{|c|}{ (b) Univariate $z_{t}, p=7$} \\
\hline$d$ & 1 & 2 & 3 & 4 & 1 & 2 & 3 & 4 \\
\hline$C(d)$ & 300.4 & 282.2 & 179.7 & 147.5 & 71.28 & 70.04 & 30.58 & 13.18 \\
\hline$d . f$. & 36 & 36 & 36 & 36 & 8 & 8 & 8 & 8 \\
\hline
\end{tabular}


Table 4: Least squares estimates and their t-ratio for the May 1993 data. The model is selected by minimum AIC and the two thresholds are -0.022574 and 0.037673 . The numbers of data points for the three regimes are 2234, 2410, and 2408, respectively.

\begin{tabular}{|c|c|c|c|c|c|c|}
\hline & \multicolumn{2}{|c|}{ Regime 1} & \multicolumn{2}{|c|}{ Regime 2} & \multicolumn{2}{|c|}{ Regime 3} \\
\hline & $f_{t}$ & $s_{t}$ & $f_{t}$ & $s_{t}$ & $f_{t}$ & $s_{t}$ \\
\hline cnst & 0.00002 & 0.00005 & 0.00000 & 0.00000 & -0.00001 & -0.00005 \\
\hline$t$ & $(1.47)$ & $(7.64)$ & $(-0.07)$ & $(0.53)$ & $(-0.74)$ & $(-6.37)$ \\
\hline$f_{t-1}$ & -0.08468 & 0.07098 & -0.03861 & 0.04037 & -0.04102 & 0.02305 \\
\hline$t$ & $(-3.83)$ & $(6.15)$ & $(-1.53)$ & (3.98) & $(-1.72)$ & $(1.96)$ \\
\hline$f_{t-2}$ & -0.00450 & 0.15899 & 0.04478 & 0.08621 & -0.02069 & 0.09898 \\
\hline$t$ & $(-0.20)$ & $(13.36)$ & $(1.85)$ & $(8.88)$ & $(-0.87)$ & $(8.45)$ \\
\hline$f_{t-3}$ & 0.02274 & 0.11911 & 0.07251 & 0.09752 & 0.00365 & 0.08455 \\
\hline$t$ & $(0.95)$ & $(9.53)$ & $(3.08)$ & $(10.32)$ & $(0.15)$ & $(7.02)$ \\
\hline$f_{t-4}$ & 0.02429 & 0.08141 & 0.01418 & 0.06827 & -0.02759 & 0.07699 \\
\hline$t$ & $(0.99)$ & $(6.35)$ & $(0.60)$ & $(7.24)$ & $(-1.13)$ & (6.37) \\
\hline$f_{t-5}$ & 0.00340 & 0.08936 & 0.01185 & 0.04831 & -0.00638 & 0.05004 \\
\hline$t$ & $(0.14)$ & $(7.10)$ & $(0.51)$ & $(5.13)$ & $(-0.26)$ & $(4.07)$ \\
\hline$f_{t-6}$ & 0.00098 & 0.07291 & 0.01251 & 0.03580 & -0.03941 & 0.02615 \\
\hline$t$ & $(0.04)$ & $(5.64)$ & $(0.54)$ & $(3.84)$ & $(-1.62)$ & $(2.18)$ \\
\hline$f_{t-7}$ & -0.00372 & 0.05201 & 0.02989 & 0.04837 & -0.02031 & 0.02293 \\
\hline$t$ & $(-0.15)$ & & & & $(-0.85)$ & \\
\hline$f_{t-8}$ & 0.00043 & 0.00954 & 0.01812 & 0.02196 & -0.04422 & 0.00462 \\
\hline$t$ & $(0.02)$ & $(0.76)$ & $(0.85)$ & $(2.57)$ & $(-1.90)$ & $(0.40)$ \\
\hline$s_{t-1}$ & 0.08419 & 0.00264 & -0.07618 & -0.05633 & 0.06664 & 0.11143 \\
\hline$t$ & $(-2.01)$ & $(0.12)$ & $(-1.70)$ & $(-3.14)$ & $(1.49)$ & $(5.05)$ \\
\hline$s_{t-2}$ & -0.05103 & 0.00256 & -0.10920 & -0.01521 & 0.04099 & -0.01179 \\
\hline$t$ & & & & & $(0.92)$ & $(-0.53)$ \\
\hline$s_{t-3}$ & 0.07275 & -0.03631 & -0.00504 & 0.01174 & -0.01948 & -0.01829 \\
\hline$t$ & $(1.65)$ & $(-1.58)$ & $(-0.12)$ & $(0.71)$ & $(-0.44)$ & $(-0.84)$ \\
\hline$s_{t-4}$ & 0.04706 & 0.01438 & 2751 & 0.01490 & 0.01646 & 0.00367 \\
\hline$t$ & $(1.03)$ & $(0.60)$ & $(0.71)$ & $(0.96)$ & $(0.37)$ & $(0.17)$ \\
\hline$s_{t-5}$ & 0.08118 & 0.02111 & 0.03943 & 0.02330 & -0.03430 & -0.00462 \\
\hline$t$ & $(1.77)$ & $(0.88)$ & $(0.97)$ & $(1.43)$ & $(-0.83)$ & $(-0.23)$ \\
\hline$s_{t-6}$ & 0.04390 & 0.04569 & 0.01690 & 0.01919 & 0.06084 & -0.00392 \\
\hline$t$ & $(0.96)$ & $(1.92)$ & $(0.44)$ & $(1.25)$ & $(1.45)$ & $(-0.19)$ \\
\hline$s_{t-7}$ & -0.03033 & 0.02051 & -0.08647 & 0.00270 & -0.00491 & 0.03597 \\
\hline$t$ & $(-0.70)$ & $(0.91)$ & $(-2.09)$ & $(0.10)$ & $(-0.13)$ & $(1.90)$ \\
\hline$s_{t-8}$ & -0.02920 & 0.03018 & 0.01887 & -0.00213 & 0.00030 & 0.02171 \\
\hline$t$ & $(-0.68)$ & $(1.34)$ & $(0.49)$ & $(-0.14)$ & $(0.01)$ & $(1.14)$ \\
\hline & 0.00024 & 0.00097 & -0.00010 & 0.00012 & 0.00025 & 0.00086 \\
\hline$t$ & $(1.34)$ & $(10.47)$ & $(-0.30)$ & $(0.86)$ & $(1.41)$ & $(9.75)$ \\
\hline
\end{tabular}


Table 5: A self-exciting threshold autoregressive model for the threshold variable $z_{t}$. The delay is 1 and the thresholds are -.017093 and .044086 .

\begin{tabular}{|c|rrrrrrrr|c|c|}
\hline regime & cnst & $z_{t-1}$ & $z_{t-2}$ & $z_{t-3}$ & $z_{t-4}$ & $z_{t-5}$ & $z_{t-6}$ & $z_{t-7}$ & $\hat{\sigma}^{2}$ & size \\
\hline 1 & -.0036 & .8109 & -.0071 & -.0185 & .0402 & -.0291 & .0274 & .0538 & .0009 & 2455 \\
$t$-ratio & -3.03 & 34.1 & -.27 & -.68 & 1.44 & -1.05 & .98 & 2.49 & & \\
\hline 2 & -.0000 & .9336 & -.0002 & .0067 & -.0160 & -.0104 & -.0033 & .0227 & .0007 & 2367 \\
$t$-ratio & -.03 & 25.56 & -.01 & .26 & -.64 & -.41 & -.13 & 1.22 & & \\
\hline 3 & .0068 & .8752 & -.0409 & -.0107 & -.0345 & .0787 & -.0296 & .0397 & .0010 & 2231 \\
$t$-ratio & 3.86 & 34.84 & -1.40 & -.36 & -1.16 & 2.63 & -1.00 & 1.85 & & \\
\hline
\end{tabular}

Table 6: Analysis for return series using a multivariate threshold model with symmetric thresholds, $p=8, q=1, r_{2} \in[.02, .085]$ and 700 grid points.

\begin{tabular}{|c|cc|r|r|rc|r|}
\hline$d$ & $\hat{r}_{1}$ & $\hat{r}_{2}$ & $\hat{A I C}$ & $d$ & $\hat{r}_{1}$ & $\hat{r}_{2}$ & $\mathrm{AIC}$ \\
\hline 1 & -.031220 & .031220 & $-1.3090 \times 10^{5}$ & 2 & -.030571 & .030571 & $-1.3082 \times 10^{5}$ \\
\hline 3 & -.029736 & .029736 & $-1.3068 \times 10^{5}$ & 4 & -.029272 & .029272 & $-1.3062 \times 10^{5}$ \\
\hline
\end{tabular}

Table 7: Results of threshold test for bivariate growth series of U.S. monthly interest rates, where $p=7$ and the degrees of freedom of the asymptotic chi-square distribution are 30 .

\begin{tabular}{|c|ccccccc|}
\hline$d$ & 1 & 2 & 3 & 4 & 5 & 6 & 7 \\
\hline & \multicolumn{7}{|c|}{$m_{o}=50$} \\
\hline$C(d)$ & 73.52 & 72.94 & 71.70 & 74.03 & 68.73 & 67.76 & 67.26 \\
\hline p-value $\times 10^{4}$ & .16 & .19 & .29 & .14 & .72 & .96 & 1.11 \\
\hline & \multicolumn{7}{|c|}{$m_{o}=100$} \\
\hline$C(d)$ & 79.28 & 63.98 & 68.40 & 72.34 & 65.21 & 61.34 & 59.01 \\
\hline p-value $\times 10^{4}$ & .03 & 2.96 & .79 & .23 & 2.06 & 6.32 & 12.11 \\
\hline
\end{tabular}

Table 8: Selection of thresholds, delay, and the number of regimes for the growth series of U.S. monthly interest rates.

\begin{tabular}{|r|r|r|rr|c|}
\hline$\hat{p}$ & $\hat{d}$ & $\hat{s}$ & $\hat{r}_{1}$ & $\hat{r}_{2}$ & AIC \\
\hline 7 & 1 & 2 & -.16977 & & -463.98 \\
\hline 7 & 4 & 2 & -.18372 & & -469.33 \\
\hline 7 & 1 & 3 & -.24930 & -.13039 & -798.79 \\
\hline 7 & 4 & 3 & -.22817 & -.10392 & -800.97 \\
\hline
\end{tabular}


Table 9: Least squares estimates and their $t$-ratios for U.S. interest-rate data. The model is selected by minimum AIC and the two thresholds are -0.228169 and -.103922 . The numbers of data points for the three regimes are 137, 139, and 126, respectively.

\begin{tabular}{|c|cc|cc|cc|}
\hline & \multicolumn{2}{|c|}{ Regime 1 } & \multicolumn{2}{c|}{ Regime 2 } & \multicolumn{2}{c|}{ Regime 3} \\
\hline & $y_{1 t}$ & $y_{2 t}$ & $y_{1 t}$ & $y_{2 t}$ & $y_{1 t}$ & $y_{2 t}$ \\
\hline cnst & 0.0017 & -0.0042 & 0.0015 & 0.0000 & -0.0034 & 0.0090 \\
$t$ & $(0.36)$ & $(-1.10)$ & $(0.34)$ & $(0.01)$ & $(-0.46)$ & $(1.75)$ \\
\hline$y_{1, t-1}$ & 0.2508 & 0.0596 & 0.4340 & 0.1949 & .0190 & 0.0182 \\
$t$ & $(2.05)$ & $(0.62)$ & $(3.60)$ & $(1.97)$ & $(0.14)$ & $(0.19)$ \\
\hline$y_{1, t-2}$ & -0.1806 & -0.1231 & -.1139 & 0.0737 & -0.0062 & 0.1590 \\
$t$ & $(-1.64)$ & $(-1.41)$ & $(-0.83)$ & $(0.66)$ & $(-0.04)$ & $(1.60)$ \\
\hline$y_{1, t-3}$ & & & -.1083 & -.1507 & -0.0495 & -0.1270 \\
$t$ & & & $(-0.70)$ & $(-1.18)$ & $(-0.35)$ & $(-1.29)$ \\
\hline$y_{1, t-4}$ & & & 0.2269 & 0.3433 & -0.0327 & 0.0010 \\
$t$ & & & $(1.31)$ & $(2.42)$ & $(-0.22)$ & $(0.01)$ \\
\hline$y_{1, t-5}$ & & & -0.1534 & -.0735 & -0.0703 & 0.0504 \\
$t$ & & & $(-0.96)$ & $(-0.56)$ & $(-0.47)$ & $(0.49)$ \\
\hline$y_{1, t-6}$ & & & -0.2262 & -0.0571 & -0.4133 & -0.3403 \\
$t$ & & & $(-1.87)$ & $(-0.58)$ & $(-2.58)$ & $(-3.09)$ \\
\hline$y_{1, t-7}$ & & & & & 0.3505 & 0.2563 \\
$t$ & & & & & $(2.18)$ & $(2.31)$ \\
\hline$y_{2, t-1}$ & 0.0741 & 0.2409 & -0.1025 & 0.2251 & 0.8361 & 0.5137 \\
$t$ & $(0.48)$ & $(1.99)$ & $(-0.66)$ & $(1.77)$ & $(4.49)$ & $(4.01)$ \\
\hline$y_{2, t-2}$ & 0.2631 & 0.0684 & 0.0600 & -0.2885 & -0.4139 & -0.5747 \\
$t$ & $(1.87)$ & $(0.61)$ & $(0.35)$ & $(-2.03)$ & $(-1.99)$ & $(-4.02)$ \\
\hline$y_{2, t-3}$ & & & 0.2857 & 0.2519 & -0.0140 & 0.0855 \\
$t$ & & & $(1.55)$ & $(1.67)$ & $(-0.07)$ & $(0.58)$ \\
\hline$y_{2, t-4}$ & & & -0.0419 & -0.2678 & -0.0610 & -0.080 \\
$t$ & & & $(-0.22)$ & $(-1.73)$ & $(-0.28)$ & $(-0.53)$ \\
\hline$y_{2, t-5}$ & & & 0.1647 & -0.0770 & 0.3672 & 0.1144 \\
$t$ & & & $(0.87)$ & $(-0.49)$ & $(1.66)$ & $(0.75)$ \\
\hline$y_{2, t-6}$ & & & 0.1054 & -0.0271 & 0.0187 & 0.0295 \\
$t$ & & & $(0.61)$ & $(-0.19)$ & $(0.09)$ & $(0.20)$ \\
\hline$y_{2, t-7}$ & & & & & -0.3117 & -0.2621 \\
$t$ & & & & & $(-1.44)$ & $(-1.76)$ \\
\hline $\mathbf{\Sigma}$ & 0.0031 & 0.0018 & 0.0025 & 0.0015 & 0.0046 & 0.0022 \\
& 0.0018 & 0.0019 & 0.0015 & 0.0017 & 0.0022 & 0.0022 \\
\hline
\end{tabular}


Table 10: Results of threshold nonlinearity test for Iceland daily river flow data, using three possible threshold variables and three bivariate AR models. The sample size is 1095, the exogenous variables used are daily precipitation lagged 1, 2 and 3 days and daily temperature lagged 0 and 1 day, and the starting point of recursive least squares is 150 , where $p$ is the AR order, $d$ is the delay and d.f. stands for degrees of freedom of the asymptotic $\chi^{2}$ distribution.

\begin{tabular}{|c|c|c|c|c|c|c|c|}
\hline & & \multicolumn{6}{|c|}{ (a) Threshold variable $z_{t}$} \\
\hline$p$ & $d$ & 0 & 1 & 2 & 3 & 4 & d.f. \\
\hline 4 & $C(d)$ & $\overline{333.2}$ & 272.4 & 164.7 & 144.7 & 143.4 & 26 \\
\hline 15 & $C(d)$ & 379.8 & 340.5 & 235.8 & 193.4 & 176.4 & 70 \\
\hline 19 & $C(d)$ & 388.1 & 343.3 & 242.5 & 203.3 & 193.3 & 86 \\
\hline & & \multicolumn{6}{|c|}{ (b) Threshold variable $y_{1 t}$} \\
\hline 4 & $C(d)$ & & 231.9 & 186.9 & 188.3 & 158.9 & 26 \\
\hline 15 & $C(d)$ & & 273.7 & 209.2 & 231.2 & 199.9 & 70 \\
\hline 19 & $C(d)$ & & 303.2 & 241.1 & 267.2 & 215.1 & 86 \\
\hline & & \multicolumn{6}{|c|}{ (c) Threshold variable $y_{2 t}$} \\
\hline 4 & $C(d)$ & & 123.2 & 86.7 & 84.2 & 53.9 & 26 \\
\hline 15 & $C(d)$ & & 179.6 & 143.0 & 149.8 & 116.0 & 70 \\
\hline 19 & $C(d)$ & & 203.5 & 177.2 & 175.2 & 137.0 & 86 \\
\hline
\end{tabular}


Table 11: Conditional least squares estimates and their $t$-ratios for a selected bivariate tworegime TAR(15) model for the Iceland river flow data. The threshold value is $-0.42394^{\circ} \mathrm{C}$ and the numbers of observations in each regime are 479 and 601, respectively. The exogenous variables are daily precipitation $x_{t}$ and temperature $z_{t}$.

\begin{tabular}{|l|rr|rr|rr|rr|}
\hline & \multicolumn{7}{|c|}{ Regime 1} & \multicolumn{3}{c|}{ Regime 2} \\
\hline & \multicolumn{2}{|c}{$y_{1 t}$} & \multicolumn{2}{|c|}{$y_{2 t}$} & \multicolumn{2}{|c|}{$y_{1 t}$} & \multicolumn{2}{|c|}{$y_{2 t}$} \\
\hline & Coef. & $t$-ratio & Coef. & $t$-ratio & Coef. & $t$-ratio & Coef. & $t$-ratio \\
\hline cnst & 7.47 & 17.55 & 1.42 & 6.48 & -0.20 & -0.20 & 1.30 & 4.59 \\
$y_{1, t-1}$ & 0.46 & 14.06 & -0.06 & -3.18 & 1.11 & 27.22 & 0.02 & 1.21 \\
$y_{1, t-2}$ & 0.08 & 2.09 & 0.03 & 1.59 & -0.44 & -7.71 & -.04 & -2.99 \\
$y_{1, t-3}$ & 0.06 & 2.35 & -0.01 & -0.42 & 0.32 & 5.34 & & \\
$y_{1, t-4}$ & 0.05 & 2.17 & 0.01 & 0.63 & -0.28 & -4.56 & & \\
$y_{1, t-5}$ & -0.08 & -3.32 & -0.02 & -1.34 & 0.18 & 2.90 & & \\
$y_{1, t-6}$ & 0.11 & 4.16 & 0.03 & 2.17 & -0.14 & -2.30 & & \\
$y_{1, t-7}$ & -0.04 & -1.63 & -0.01 & -0.57 & 0.07 & 1.22 & & \\
$y_{1, t-8}$ & -0.01 & -0.37 & -0.01 & -0.83 & 0.02 & 0.41 & & \\
$y_{1, t-9}$ & 0.02 & 0.87 & 0.02 & 2.09 & -0.02 & -0.63 & & \\
$y_{1, t-10}$ & -0.05 & -1.70 & & & & & & \\
$y_{1, t-11}$ & 0.05 & 2.06 & & & & & & \\
$y_{1, t-12}$ & 0.01 & 0.36 & & & & & & \\
$y_{1, t-13}$ & 0.04 & 1.54 & & & & & & \\
$y_{1, t-14}$ & -0.07 & -2.91 & & & & & & \\
$y_{1, t-15}$ & 0.05 & 2.70 & & & & & & \\
$y_{2, t-1}$ & 0.39 & 6.77 & 0.80 & 24.70 & 0.90 & 7.12 & 1.26 & 28.39 \\
$y_{2, t-2}$ & & & -0.18 & -4.73 & -1.09 & -5.11 & -0.67 & -8.99 \\
$y_{2, t-3}$ & & & 0.09 & 2.80 & 0.20 & 0.87 & 0.25 & 3.06 \\
$y_{2, t-4}$ & & & 0.03 & 0.82 & 0.51 & 2.20 & 0.16 & 1.98 \\
$y_{2, t-5}$ & & & -0.02 & -0.58 & -0.17 & -0.76 & -0.00 & -0.05 \\
$y_{2, t-6}$ & & & 0.02 & 0.54 & 0.11 & 0.57 & -0.03 & -0.49 \\
$y_{2, t-7}$ & & & 0.00 & 0.03 & 0.06 & 0.31 & 0.16 & 2.26 \\
$y_{2, t-8}$ & & & 0.02 & 0.60 & -0.57 & -2.88 & -0.30 & -4.43 \\
$y_{2, t-9}$ & & & -0.02 & -0.41 & 0.52 & 3.91 & 0.17 & 4.10 \\
$y_{2, t-10}$ & & & -0.04 & -1.12 & & & & \\
$y_{2, t-11}$ & & & 0.05 & 1.46 & & & & \\
$y_{2, t-12}$ & & & 0.01 & 0.42 & & & & \\
$y_{2, t-13}$ & & & -0.08 & -2.19 & & & & \\
$y_{2, t-14}$ & & & 0.09 & 4.08 & & & & \\
$x_{t-1}$ & 0.06 & 4.59 & 0.01 & 1.84 & 0.47 & 10.89 & 0.09 & \\
$x_{t-2}$ & -0.03 & -2.49 & -0.00 & -0.11 & -0.23 & -4.71 & -0.06 & -3.77 \\
$x_{t-3}$ & 0.04 & 3.36 & -0.01 & -2.03 & & & 0.05 & 2.96 \\
$z_{t}$ & 0.03 & 1.63 & 0.01 & 0.93 & 0.88 & 4.78 & & \\
$z_{t-1}$ & -0.02 & -1.08 & -0.02 & -1.67 & 0.30 & 1.92 & & \\
\hline
\end{tabular}




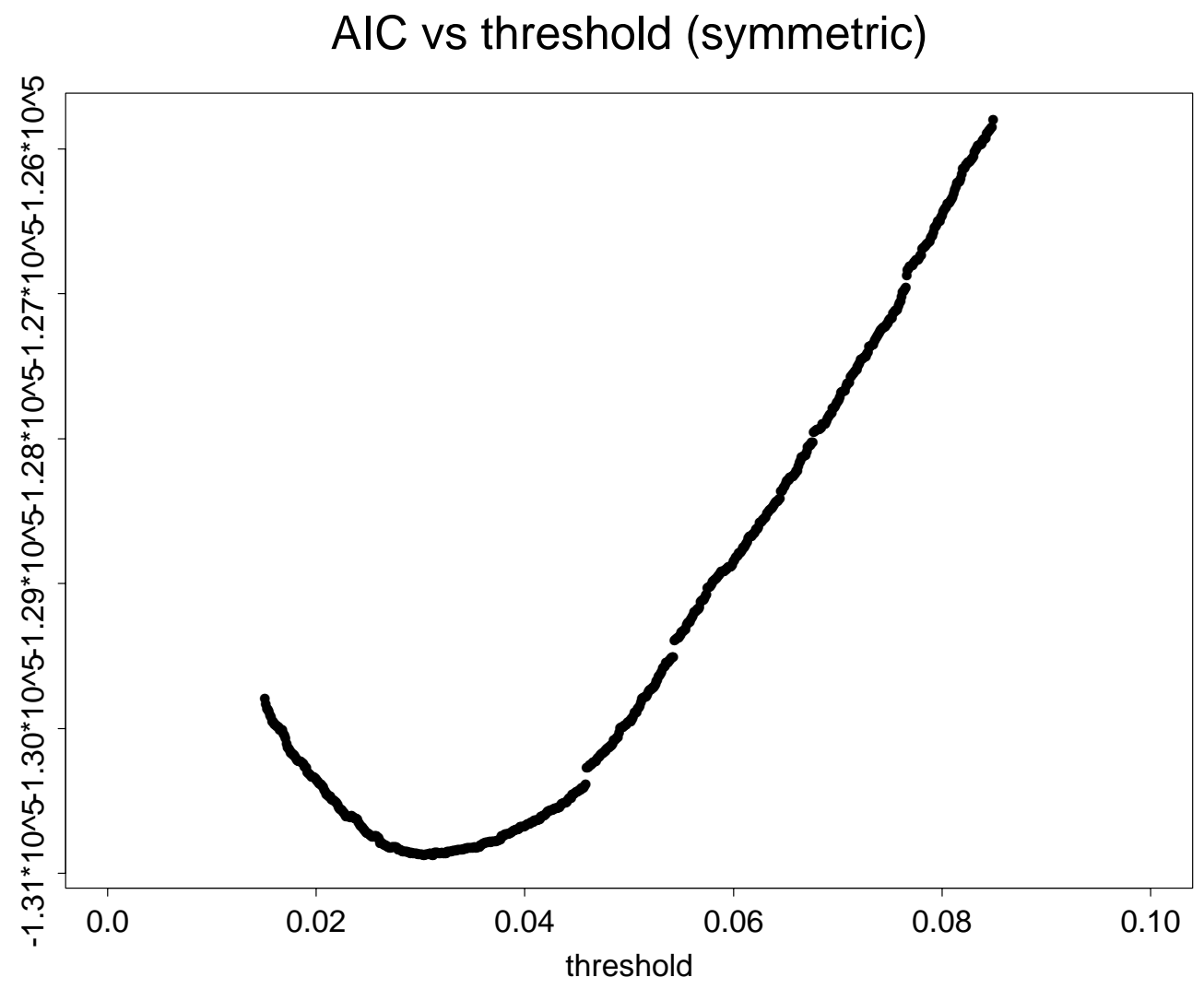

Figure 2: AIC versus symmetric threshold 

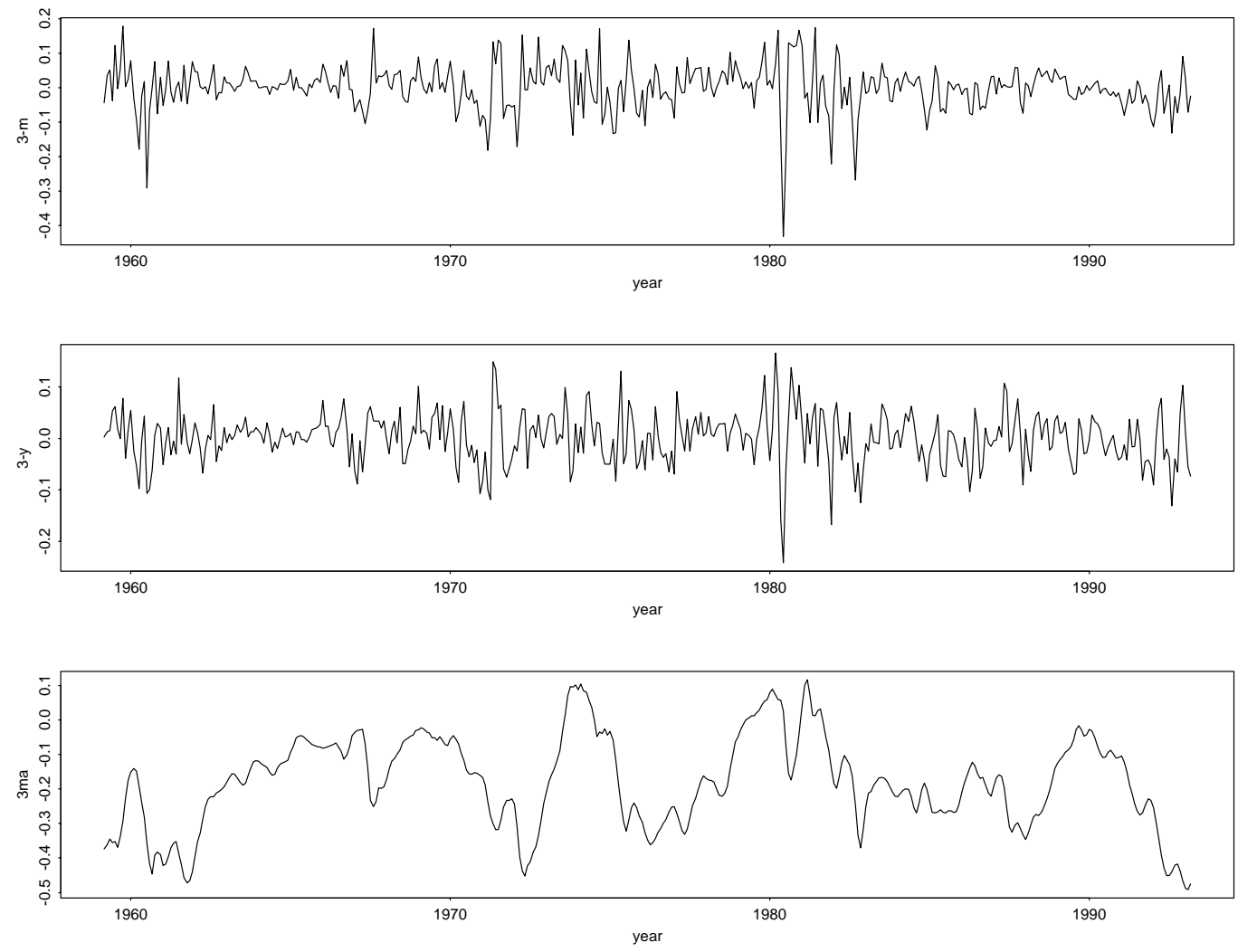

Figure 3: Time plots of growth series of U.S. monthly interest rates from 1959.2 to 1993.2. (a) 3-month Treasury Bills, (b) 3-year Treasury Notes, and (c) 3-month moving average of spread in logged interest rates. 

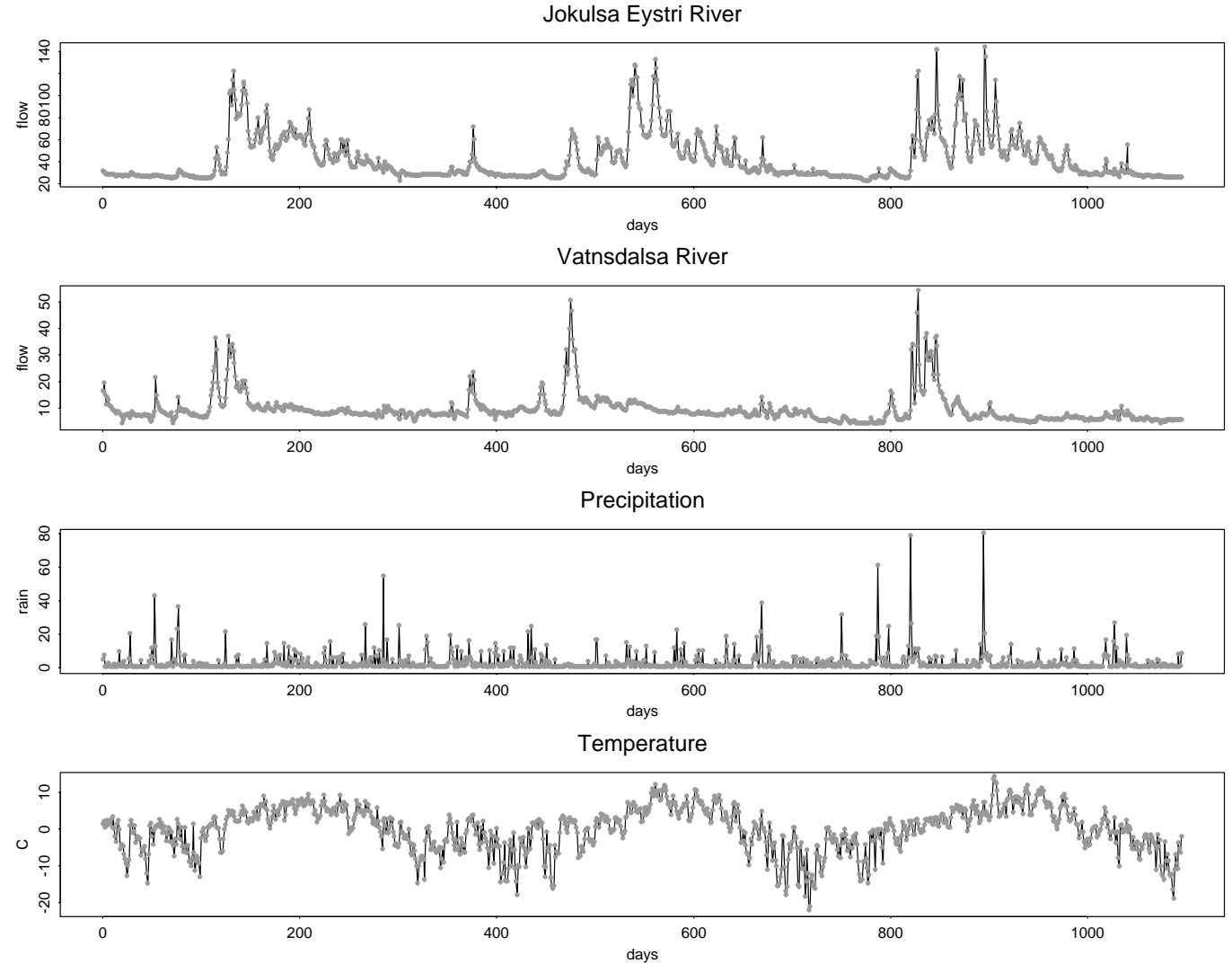

Figure 4: Time plots of daily flow of Jökulsá Eystri River and Vatnsdalsá River of Iceland in 1972-1974. The exogenous variables are daily precipitation and temperature measured at the Hveravellir meteorological station. 


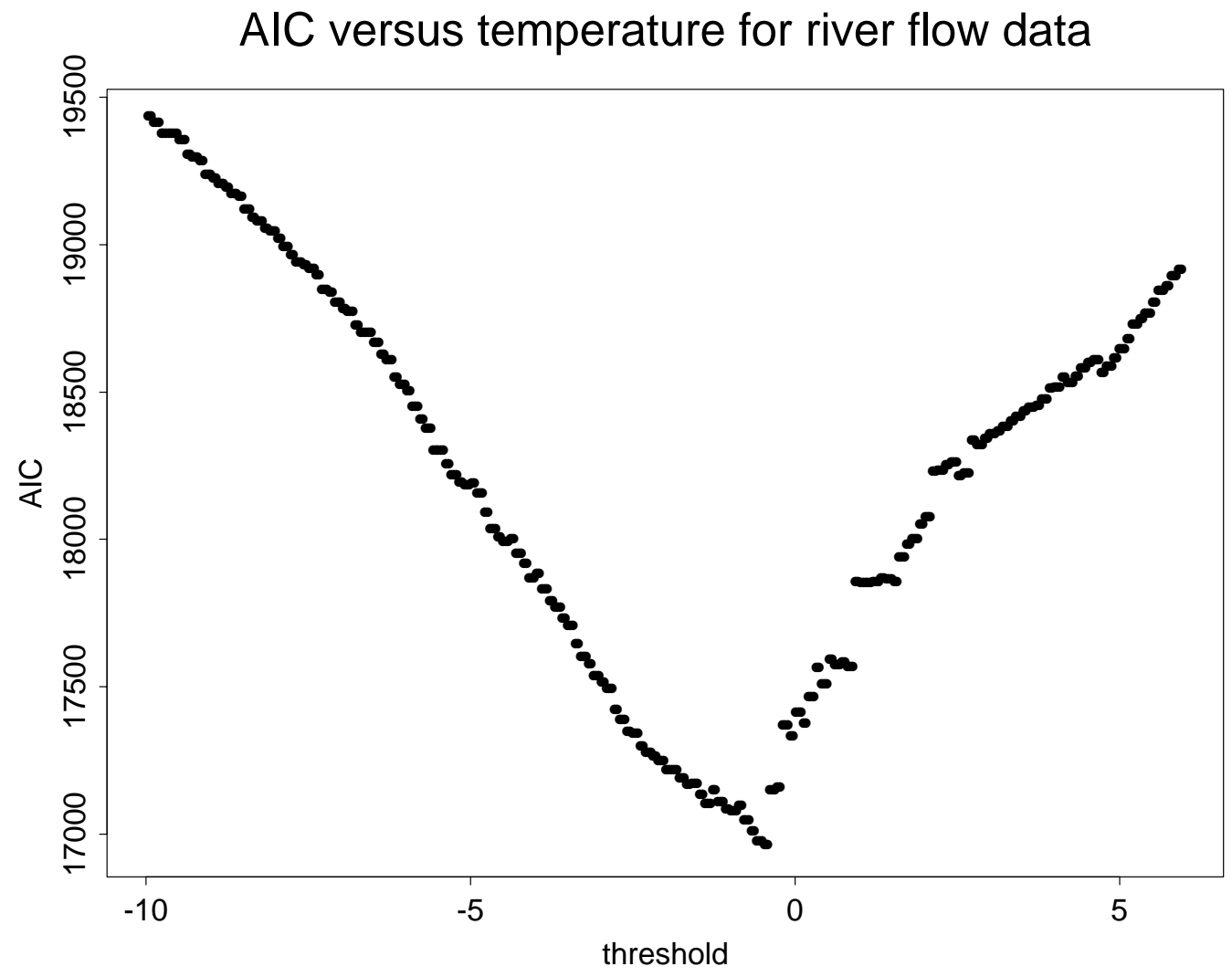

Figure 5: Scatterplot of AIC versus temperature for Iceland river flow data using a tworegime bivariate $\operatorname{TAR}(15)$ model. 